Article

\title{
Three-Dimensional Modeling of a Robotic Fish Based on Real Carp Locomotion
}

\author{
Gonca Ozmen Koca ${ }^{1, *}$, Cafer Bal ${ }^{1}$, Deniz Korkmaz ${ }^{2}$ (D), Mustafa Can Bingol ${ }^{1}$, Mustafa Ay ${ }^{1}$ (D), \\ Zuhtu Hakan Akpolat ${ }^{1}$ and Seda Yetkin ${ }^{3}$ \\ 1 Department of Mechatronics Engineering, University of Firat, Elazig 23119, Turkey; \\ caferbal@gmail.com (C.B.); mustafacanbingol@gmail.com (M.C.B.); mustafaay023@gmail.com (M.A.); \\ z.h.akpolat@gmail.com (Z.H.A.) \\ 2 Department of Electrical and Electronics Engineering, University of Firat, Elazig 23119, Turkey; \\ denizkorkmaz17@gmail.com \\ 3 Department of Electronics and Automation, University of Bitlis Eren, Bitlis 13000, Turkey; \\ syetkin@beu.edu.tr \\ * Correspondence: gonca.ozmen@gmail.com; Tel.: +90-424-237-0000
}

Received: 9 November 2017; Accepted: 22 January 2018; Published: 26 January 2018

\begin{abstract}
This work focuses on developing a complete non-linear dynamic model comprising entirely kinematic and hydrodynamic effects of Carangiform locomotion based on the Lagrange approach by adapting the parameters and behaviors of a real carp. In order to imitate biological features, swimming patterns of a real carp for forward, turning and up-down motions are analyzed by using the Kineova 8.20 software. The proportional optimum link lengths according to actual size, swimming speed, flapping frequency, proportional physical parameters and different swimming motions of the real carp are investigated with the designed robotic fish model. Three-dimensional (3D) locomotion is evaluated by tracking two trajectories in a MATLAB environment. A Reaching Law Control (RLC) approach for inner loop (Euler angles-speed control) and a guidance system for the outer loop (orientation control) are proposed to provide an effective closed-loop control performance. In order to illustrate the 3D performance of the proposed closed loop control system in a virtual reality platform, the designed robotic fish model is also implemented using the Virtual Reality Modeling Language (VRML). Simulation and experimental analysis show that the proposed model gives us significant key solutions to design a fish-like robotic prototype.
\end{abstract}

Keywords: dynamic model; robotic fish; trajectory tracking; biomimetic modeling; fish-like motion

\section{Introduction}

In recent years, biologically inspired behavior-based systems have been more and more popular topic in underwater vehicles. The goal of the bio-inspired approach is to adapt the biological features of underwater creatures such as fish to Autonomous Underwater Vehicle (AUV) designs and also imitate the aquatic locomotion abilities of them. Biomimetic modeling also provides enhanced performance and increased efficiency, maneuverability and acceleration for novel AUV designs [1-5]. As it is known, many kinds of fish perform high-efficiently locomotion and maneuvering in the water. Propulsion efficiencies of the rotary propeller AUVs are limited below $70 \%$, while the swimming mechanism of a real fish is $20 \%$ more efficient than rotary propellers [6]. In addition, the turning radius of propellers is big and speed performances are low. Therefore, this kind of propulsion is more noisy and ineffective than bio-inspired systems [6,7]. These advantages have great benefit for marine applications and fish-like robots have evolved to provide versatile solutions for a wide variety of underwater applications, such as undersea investigation, pollution detection, deep sea monitoring and mapping, military operations and protections, etc. [8-11]. 
An elaborate observation of the whole biological structure of a fish is necessary to identify the principles of a bio-inspired approach to the fish. In this way, significant limitations and constraints of any biological feature are determined before modeling the robotic fish. A bio-inspired robotic fish is defined as an aquatic vehicle, which is propelled by undulatory or oscillatory body motions $[10,12]$. In nature, most fish exhibit various swimming behaviors by bending their bodies and / or their caudal fins (BCF). Alternatively, some fish use their median and/or pectoral fins (MPF) [13]. Commonly, more than $85 \%$ of fish swim by BCF locomotion and on average $15 \%$ of fish swim in the world by MPF. Carangiform locomotion, the most common type of $\mathrm{BCF}$, generates undulatory swimming patterns, high speed performance and low noise [11,13]. Thus, biomimetic Carangiform design is an appropriate solution for AUVs [14-17].

There have been some studies about robotic fish modeling in the literature. The first mathematical models including fish swimming were proposed by Wu and Lighthill in 1960s. Wu suggested simplified two-dimensional waving plate theory and Lighthill defined the body-traveling wave, which takes a sinusoidal form of undulatory motion $[1,18]$. Sfakiotakis explained the fish-swimming modes for oscillatory and undulatory motions of fins and bodies [14]. McIsaac and Ostrowski established a model for Anguilliform locomotion to give swimming gaits [19], while Morgansen et al. derived 3D models for pectoral fins and tails [20]. Also, Yu et al. applied the Central Pattern Generator (CPG)-based locomotor controller to their dynamic model. CPG is a neural circuit diagram inspired from biological musculoskeletal systems to perform gait generation of bio-inspired robots [21-23]. Liu and Hu built a simulator for the autonomous Carangiform motion of a four-joint robotic fish, but up-down motions were not modeled [24]. Yu et al. also modeled up-down motions [25,26]. Unlike these, Zhou et al. presented a dynamic backward analysis for a biomimetic Carangiform robotic fish [27]. Furthermore, Nakashima and Ohgishi presented a dynamic model of the double-jointed robotic fish to examine self-exciting conditions and propulsive characteristics [28]. Kim et al. presented the four-link motion mechanism of fish [29]. Masoomi and colleagues modeled a four degrees of freedom (4-DoF) mathematical model including hydrodynamic forces inspired by tuna fish [10]. A multi-DoF robotic fish model was suggested by Suebsaiprom and Lin to determine the position and orientation of the rigid body. They used a state feedback controller in the simulation $[15,30]$. According to the above literature survey, a 3D dynamic model including all kinematic and hydrodynamic effects by analyzing realistic parameters of a real fish would be a significant reference while developing a realistic prototype.

The contribution of this paper is to develop a complete dynamic model including kinematic and hydrodynamic effects of Carangiform locomotion based on the Lagrange approach by adapting the parameters and behaviors obtained from real carp. This model provides appropriate solutions for biomimetic design of fish-like motions with 3D carp gait patterns unlike the dynamic robotic fish models available in the literature with non-realistic parameters. In order to mimic real fish-swimming abilities, the designed robotic fish model has been focused on the proportional optimum link lengths according to actual size, swimming speed, flapping frequency, proportional physical parameters and different swimming motions of the real carp. 3D locomotion is evaluated by applying various trajectories in different MATLAB environments. The closed loop performance is provided by using the Reaching Law Control (RLC) approach in the inner loop and orientation control is also achieved with a guidance system in the outer loop. These simulations and experimental analyses show that the proposed model gives us significant key solutions to design a fish-like robotic prototype.

The rest of this paper is organized as follows: in the next section, Carangiform locomotion is described and a complete dynamic model, including kinematic and hydrodynamic effects, is given. Then the maneuverability of the robotic fish model is performed and simulation results are given. Finally, conclusions are presented.

\section{Three Dimensional Dynamic Model and Motion Equations of the Robotic Fish}

The fish model analyzed in this study is inspired by a Carangiform-mode fish based on the BCF locomotion. A Carangiform mode exhibits more significant swimming actions such as fast start, 
rapid turning, C-shape turning, cruise swimming and high accelerations [14]. A Carangiform fish swims on the lateral sinusoidal motion, which increases the amplitude from nose to caudal fin in a spine. This kinematic motion can be described using the body-traveling wave function, suggested by Lighthill [18,31]. However, this function only includes the simple kinematic equality for the fish tail and there is no whole dynamic effect to understand the fish locomotion. Based on the biological features of Carangiform locomotion, the non-linear dynamic model of the robotic fish should be derived, including 6-DoF motion. This study focuses on deriving a complete non-linear mathematical model of a Carangiform robotic fish by analyzing real carp locomotion. The dynamic model is performed by using realistic parameters from 3D swimming patterns of the carp. The proposed non-linear mathematical model of the robotic fish includes the body and tail kinematics, hydrodynamic effects acting on each link and rigid body motions. This model behaves like a real fish with complete non-linear derivations. In this way, biomimetic modeling of the robotic fish prototype can be easily implemented in future work.

\subsection{Carp Locomotion}

In this study, forward, turning and ascending-descending swimming patterns are investigated with top- and side-view cameras for 3D records in a $120 \mathrm{~cm}$ length, $80 \mathrm{~cm}$ width and $60 \mathrm{~cm}$ depth test tank to analyze the maneuverability of real carp. The resolution of the high-definition video camera is $1920 \times 1080 \mathrm{p}$, the data rate is $19,949 \mathrm{~kb} / \mathrm{s}$, the total bit rate is $20,205 \mathrm{~kb} / \mathrm{s}$ and frame rate is $29 \mathrm{fps}$, respectively. Stabilized swimming over a distance of $60 \mathrm{~cm}$ is selected for testing. Snapshots are also captured and examined by using the Kinovea 8.20 software. The sampling time is set to $0.05 \mathrm{~s}$.

The main idea for kinematic modeling of the robotic fish is to assume the robotic fish to have a multi-link mechanism. The equivalent model of the robotic fish and description of the one link are given in Figure 1.

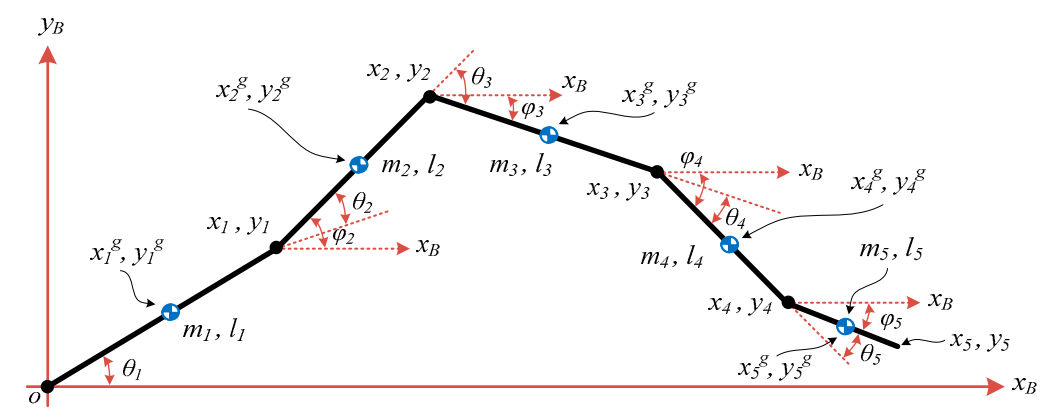

(a)

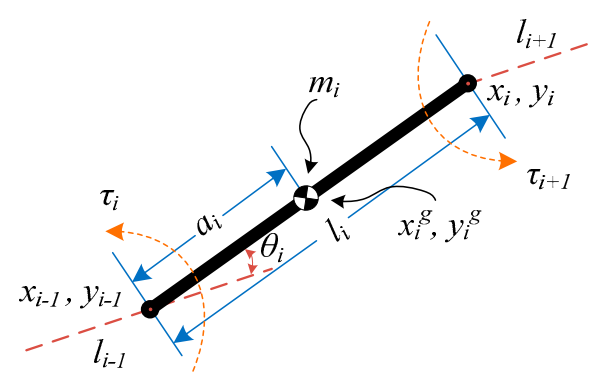

(b)

Figure 1. Kinematic scheme of the robotic fish: (a) Equivalent model; (b) Description of the one link.

The designed robotic fish consists of five links. We will define $\left[l_{1}, \ldots, l_{i}\right] \rightarrow(i=1,2, \ldots, N)$ as one body link, three active tail links and one passive oscillating caudal fin link. $N=5$ is the number of links. $\theta_{1}$ is the angle of head during swimming, $\theta_{k}(k=2,3,4)$ is the each active link angle of the 
propulsion mechanism and $\theta_{5}$ is angle of the caudal fin. $m_{1}$ is the body mass and each link mass of the propulsion mechanism equals from $m_{2}$ to $m_{5} .\left(x_{i}^{g}, y_{i}^{g}\right)$ and $\left(x_{i}, y_{i}\right)$ are link coordinates of the center of mass and end points of the link coordinates, respectively. Also, $\varphi_{i}=\theta_{i}+\theta_{i-1}$ is the orientation angle of the joints in the horizontal plane. It can be seen from Figure $2 b$ that each link is homogeneous and the middle points of the links are regarded as the center of mass. $a_{i}$ is the distance from joint to center of the link mass and $\tau_{i}$ is the turning moment of the each joint.

Motion of a real fish depends on the body-traveling wave. In order to imitate the body-traveling wave, there are two general methods: sine-based joint location or intersection methods [19,32], and CPGs [21,33]. In this paper, the improved intersection method with the Big Bang-Big Crunch optimization algorithm, which is also used in our previous work [32], is applied to the link points of the real carp. In this method, locations of links are determined within a scanning area to achieve the minimum error of the fitting body-travelling wave. Thus, optimization criteria is chosen as error of the envelop area with optimum link lengths [32,34]. In the analysis of the carp, five points are defined to verify optimized link lengths on forward and turning patterns obtained from the top view of the carp. The locations of the points and optimized link lengths on forward and the turning patterns are shown in Figure 2.

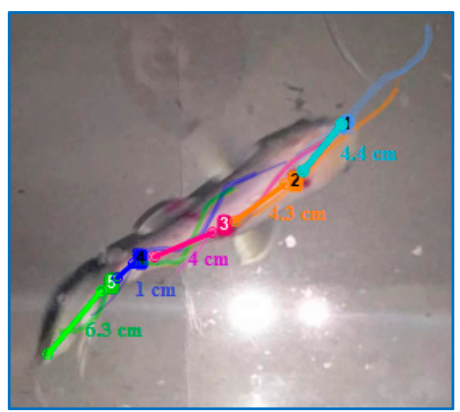

(a)

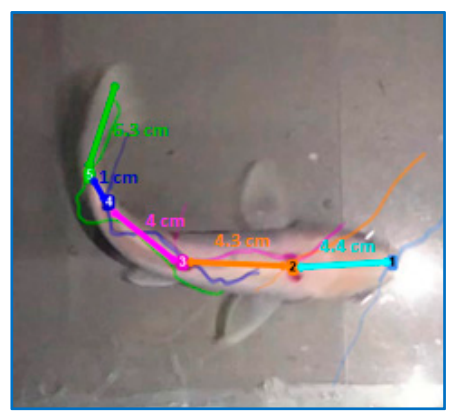

(b)

Figure 2. Trajectory study of two swimming patterns of carp. The fish is simplified as a 5-joint linkage represented by different colors. The light color curves indicate a trajectory of the corresponding joint within a period of swimming pattern: (a) Forward swimming pattern, period 350 ms; (b) Turning swimming pattern, period $550 \mathrm{~ms}$.

In the experiments, five points are marked on the real fish in order to define link locations according to optimized link lengths of the robotic fish. Locations of the points are tested by applying links between every two points to ensure a minimum area.

As seen from Figure 2, light blue line is the anterior body, orange, pink and deep blue lines are active links and the green line is the passive caudal fin, respectively.

\subsection{Swimming Motion of the Robotic Fish}

Motions of the carp obtained from experimental analysis according to all joints for forward and turning swimming modes are given in Figure 3.

The average speed of a carp ranges from 0 to $2.5 \mathrm{~m} / \mathrm{s}$ in the ichthyology. In the experimental studies, speeds of forward swimming are recorded for some different ranges from $0.105 \mathrm{~m} / \mathrm{s}, 0.125 \mathrm{~m} / \mathrm{s}$, $0.144 \mathrm{~m} / \mathrm{s}, 0.233 \mathrm{~m} / \mathrm{s}$ to $0.41 \mathrm{~m} / \mathrm{s}$ and turning speed is obtained as $0.24 \mathrm{~m} / \mathrm{s}$, approximately. In order to determine flapping frequency of the carp, motion of the caudal fin is analyzed as shown in Figure 4 during different periods. The average recorded flapping frequencies of the carp are measured as $1.818 \mathrm{~Hz}, 2 \mathrm{~Hz}, 2.439 \mathrm{~Hz}$ and $2.857 \mathrm{~Hz}$, approximately. In order to calculate one sample flapping frequency for $2.857 \mathrm{~Hz}$, minimum and maximum angles of the caudal fin from the center of gravity are measured and time of the fin motion is determined. In this sample, motion time for one period is measured as $350 \mathrm{~ms}$. 


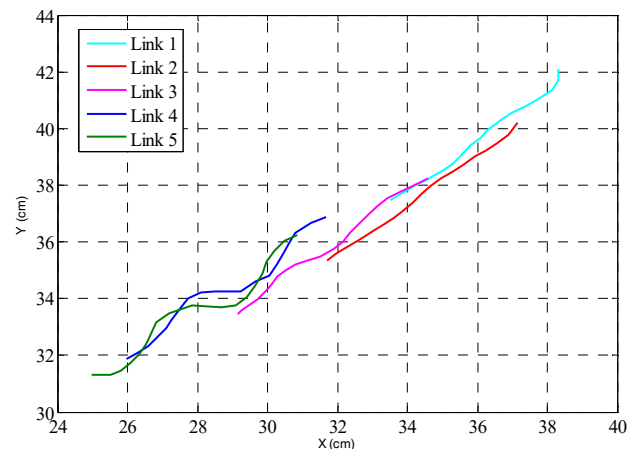

(a)

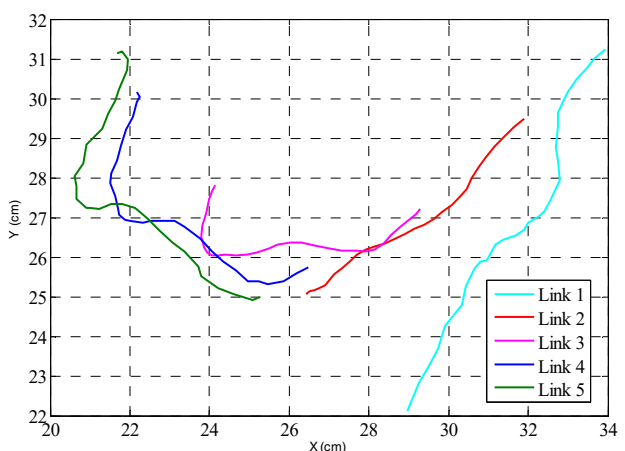

(b)

Figure 3. Path tracking of the all links: (a) Forward swimming pattern; (b) Turning swimming pattern.

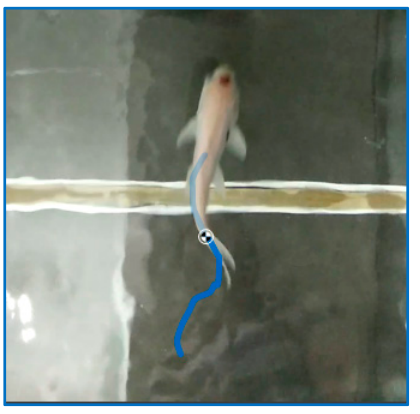

(a)
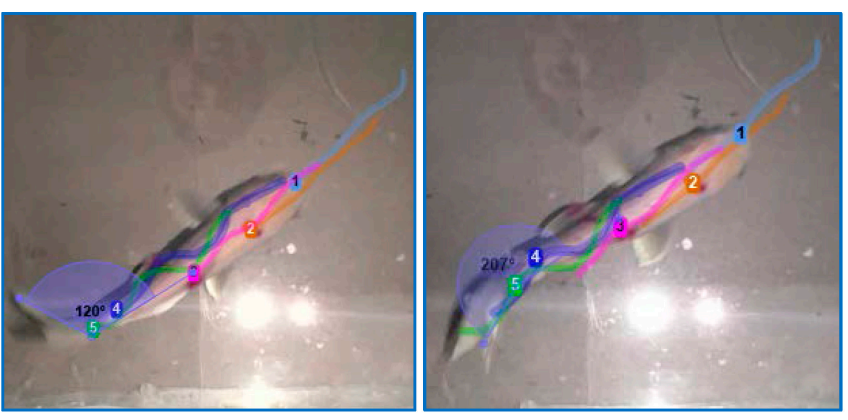

(b)

Figure 4. One period of the tail: (a) Trace of the travelling wave for $350 \mathrm{~ms}$; (b) Caudal fin angles for forward swimming pattern: $\theta_{5}=[\pi-0.6 \pi, \pi-1.15 \pi]$.

Angles of the active links are also shown in Figure 5 for forward and turning swimming modes during one period.

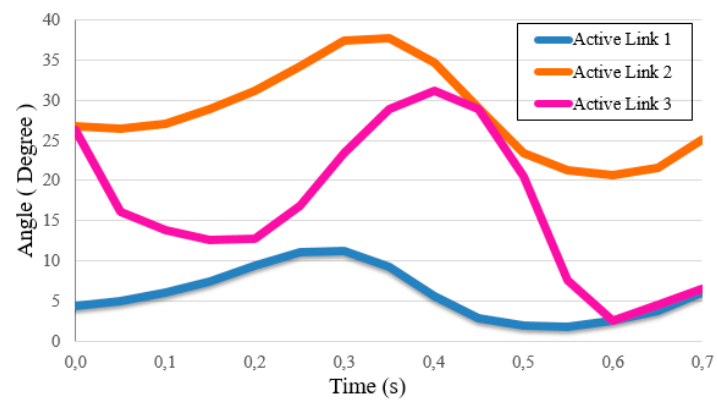

(a)

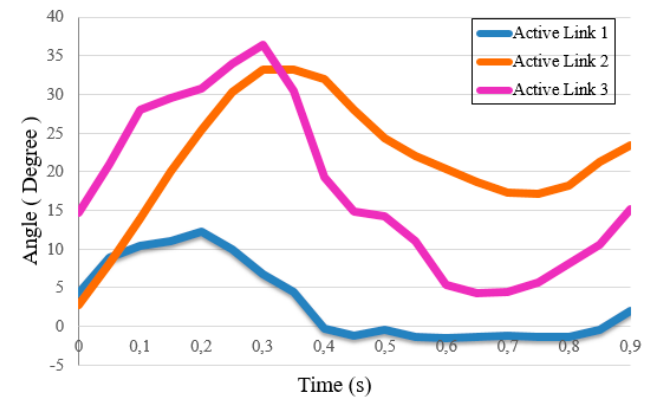

(b)

Figure 5. Real fish angles according to the active links: (a) Forward swimming mode; (b) Turning swimming mode.

Motion of the robotic fish is realized by the powerful spine that generates the lateral sinusoidal undulations, while the anterior body is relatively rigid and determines the swimming direction. Active tail links are actuated by servomotors and the passive caudal fin is connected to the fourth link with a peduncle. It is noted that the thrust force is generated by the caudal fin.

In order to obtain real fish-like motions, the dynamic model of the robotic fish is derived by using following second order form:

$$
M\left(\theta_{i}\right) \ddot{\theta}+C\left(\theta_{i}, \dot{\theta}_{i}\right) \dot{\theta}_{i}+B\left(\dot{\theta}_{i}\right)+K\left(\theta_{i}\right)=\tau_{i}
$$


$M\left(\theta_{i}\right)$ is the moment of inertia, $C\left(\theta_{i}, \dot{\theta}_{i}\right)$ is the Coriolis matrix, $B\left(\dot{\theta}_{i}\right)$ is the damping coefficient matrix, $K\left(\theta_{i}\right)$ is the spring coefficient matrix and $\tau_{i}$ is the force vector that includes hydrodynamic forces.

\subsection{Hydrodynamic Forces Acting on the Tail}

As shown in Figure 6, hydrodynamic forces depend on the tail motion and there are five main hydrodynamic forces acting on the tail. Inertial fluid force is $F_{V}$, lift force is $F_{J}$, thrust force is $F_{F}$, lateral force is $F_{C}$ and drag force is $F_{D}$.

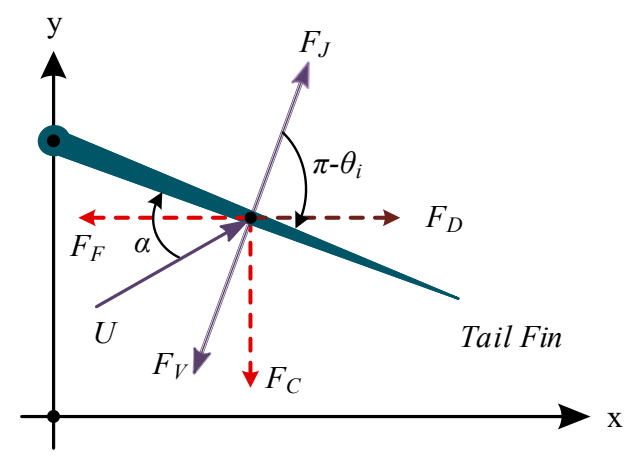

Figure 6. Hydrodynamic force distributions acting on the tail.

It is considered that tail fin of the robotic fish flaps in constant flow representing by $U_{m}$ so that the inertial and lift forces influence the caudal fin. Afterwards, thrust force and lateral force components are calculated by using inertial fluid and lift forces. The value of constant flow is determined as $0.08 \mathrm{~m} / \mathrm{s}$ to reduce the turbulent effects [35]. According to Figure $6, F_{V}$ is a proportional force to the acceleration and it is calculated by Equation (2) and the lift force is also vertical to the flow and it is defined as Equation (3):

$$
\begin{gathered}
F_{V}=\pi \rho L C^{2} \dot{U} \sin (\alpha)+\pi \rho L C^{2} U \dot{\alpha} \cos (\alpha) \\
F_{J}=2 \pi \rho L C U^{2} \sin (\alpha) \cos (\alpha)
\end{gathered}
$$

here, the chord length is $2 C$, the span of the caudal fin is $L$, density of the water is $\rho$, relative speed at the center of caudal fin is $U$ and the angle of attack is $\alpha$. Relative speed and angle of attack variables are time dependent and the first derivative of these should be obtained, smoothly. These forces are separated into the thrust force on the $\mathrm{x}$-axis and lateral force on the $\mathrm{y}$-axis components:

$$
\begin{aligned}
& F_{F}=\left(F_{V}-F_{J}\right) \sin \left(2 \pi-\sum_{i=1}^{N} \theta_{i}\right) \\
& F_{C}=\left(F_{V}-F_{J}\right) \cos \left(2 \pi-\sum_{i=1}^{N} \theta_{i}\right)
\end{aligned}
$$

If motion of the robotic fish is considered in the x-axis direction, the relative speed on the center of caudal fin in the y-axis direction is expressed by the following equation:

$$
\begin{aligned}
u_{f}= & l_{1} \dot{\theta}_{1} \cos \left(\theta_{1}\right)+l_{2}\left(\dot{\theta}_{1}+\dot{\theta}_{2}\right) \cos \left(\theta_{1}+\theta_{2}\right) \\
& +l_{3}\left(\dot{\theta}_{1}+\dot{\theta}_{2}+\dot{\theta}_{3}\right) \cos \left(\theta_{1}+\theta_{2}+\theta_{3}\right) \\
& +l_{4}\left(\dot{\theta}_{1}+\dot{\theta}_{2}+\dot{\theta}_{3}+\dot{\theta}_{4}\right) \cos \left(\theta_{1}+\theta_{2}+\theta_{3}+\theta_{4}\right) \\
& +a_{5}\left(\dot{\theta}_{1}+\dot{\theta}_{2}+\dot{\theta}_{3}+\dot{\theta}_{4}+\dot{\theta}_{5}\right) \cos \left(\theta_{1}+\theta_{2}+\theta_{3}+\theta_{4}+\theta_{5}\right)
\end{aligned}
$$

Relative speed on the center of caudal fin $U$ is determined by $\sqrt{U_{m}{ }^{2}+u_{f}{ }^{2}}$, because $U_{m}$ and $u_{f}$ are perpendicular to each other. The thrust force $F_{F}$ pushes the robotic fish in the forward direction. 
The drag force $F_{D}$ acts on the fish body in the flow direction by the friction. The definition of the drag force is presented in Equation (7):

$$
F_{D}=\frac{1}{2} \rho C_{D} V_{f}^{2} S
$$

where, $C_{D}$ is the drag coefficient, $V_{f}$ is the relative speed of the robotic fish and $S$ is the area of the main body. The head of the robotic fish is assumed as conic and the value of drag coefficient is defined as 0.5 . Both hydrodynamic and external forces act on the robotic fish tail. These forces are called with $\tau_{i}=\left[\tau_{1}, \tau_{2}, \tau_{3}, \tau_{4}, \tau_{5}\right]^{T}$ which affect to the $i$ th link, and can be expressed by:

$$
\text { if }\left\{\left.\begin{array}{l}
\text { active_link } \rightarrow \tau_{i}=F_{C} l_{i} \cos \left(\sum_{i=1}^{N} \theta_{i}\right)-F_{F} l_{i} \sin \left(\sum_{i=1}^{N} \theta_{i}\right)+T_{i-1}^{u} \\
\text { passive_link } \rightarrow \tau_{i}=F_{C} l_{i} \cos \left(\sum_{i=1}^{N} \theta_{i}\right)-F_{F} l_{i} \sin \left(\sum_{i=1}^{N} \theta_{i}\right)
\end{array}\right|_{\text {last_link } \rightarrow l_{i}=a_{i}}\right.
$$

The designed robotic fish is driven by three active joints that generate the thrust force. These joints are actuated by three DC servo motors with input torques $T_{i}^{u}=A_{\max } \sin \left(2 \pi f_{T} t \pm \Delta_{i}\right) . A_{\max }$ is the amplitude of input torque, $f_{T}$ is the frequency of input torque and $\pm \Delta_{i}$ is the phase angle between the two input torques. By using Lagrange's equation, the dynamic model of the robotic fish is described briefly in Equation (9):

$$
\left[\begin{array}{lllll}
M_{11} & M_{12} & M_{13} & M_{14} & M_{15} \\
M_{21} & M_{22} & M_{23} & M_{24} & M_{25} \\
M_{31} & M_{32} & M_{33} & M_{34} & M_{35} \\
M_{41} & M_{42} & M_{43} & M_{44} & M_{45} \\
M_{51} & M_{52} & M_{53} & M_{54} & M_{55}
\end{array}\right]\left[\begin{array}{l}
\ddot{\theta}_{1} \\
\ddot{\theta}_{2} \\
\ddot{\theta}_{3} \\
\ddot{\theta}_{4} \\
\ddot{\theta}_{5}
\end{array}\right]=\left[\begin{array}{c}
N_{1} \\
N_{2} \\
N_{3} \\
N_{4} \\
N_{5}
\end{array}\right]
$$

$M_{i j}$ is the moment element of the inertia matrix and each value of $\theta_{i}$ can be obtained from Equation (9).

\subsection{Modeling of the Up-Down Motion Mechanism}

In nature, up-down motions such as ascending and descending are another important criterion to exhibit high swimming performance. In order to generate ascending and descending motions, a fish changes its center of mass (COM) position and uses the air bladder. Figure 7 gives the descending pattern of the carp during $1.05 \mathrm{~s}$. In this analysis, pitch angle of the carp is measured as $35^{\circ}$. This performance is the high motion ability for a real fish and similar values of this angle are validated by using the derived model.

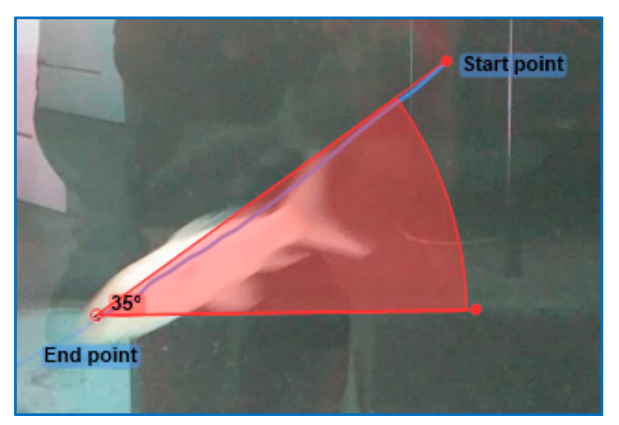

Figure 7. Descending pattern of the carp during $1.05 \mathrm{~s}$.

In Figure 8a,b, the model of the up-down motion mechanism and flow chart, which represents the working principle of the mechanism, are given, respectively. An up-down mechanism is designed and modeled by using the Lagrange approach to generate and control pitch and roll motions. 
This mechanism is placed inside the anterior robotic fish body. While the robotic fish is swimming under water, swimming speed is not zero and the depth position of the robotic fish in the vertical plane can be controlled. Control of the orientation angles are performed by changing COM position. There are two masses $\left(m_{x}, m_{y}\right)$ moving on the shaft. Shafts are connected to the pulley sets with DC motors. By moving the masses along the $\mathrm{x}$ and $\mathrm{y}$-axis, the up-down mechanism changes the pitch and roll angles.

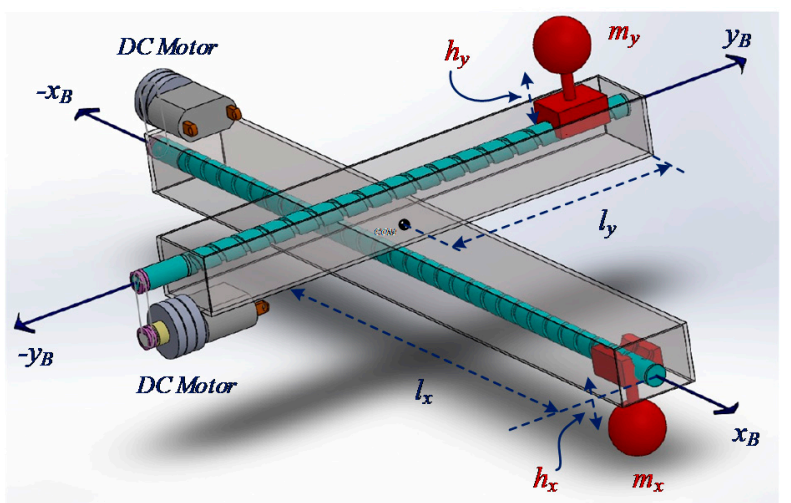

(a)

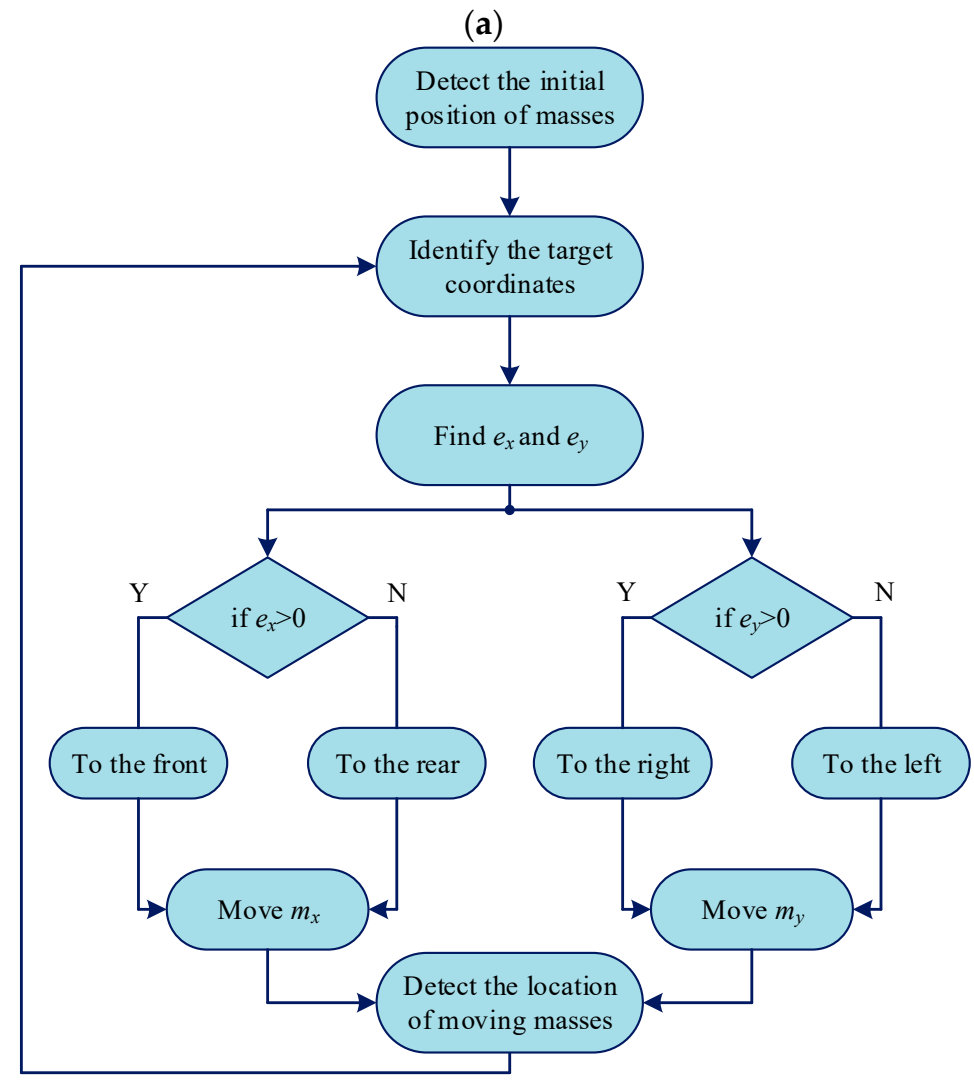

(b)

Figure 8. (a) Model of the up-down motion mechanism; (b) Flow chart of the up-down motion.

When mass positions change, pitch and roll torques are generated. These mentioned torques are considered in Equations (10) and (11), respectively.

$$
\begin{gathered}
\tau_{x}=g\left(\left(l_{x} \sin (\phi) \sin (\theta)-h_{x} \sin (\phi) \cos (\theta)\right) m_{x}+\left(l_{y} \cos (\phi)-h_{y} \sin (\phi) \cos (\theta)\right) m_{y}\right) \\
\tau_{y}=g\left(\left(l_{x} \cos (\theta)-h_{x} \sin (\theta)\right) m_{x}+\left(h_{y} \sin (\theta)\right) m_{y}\right)
\end{gathered}
$$


Here, $g$ is the acceleration of the gravity, $\left(l_{x}, l_{y}\right)$ are the distance from the origin and $\left(h_{x}, h_{y}\right)$ are the heights from the axis.

\subsection{Three Dimensional Model Equations of Motion}

Based on the ichthyology of carp, a multi-link and autonomous-swimming biomimetic robotic fish prototype is designed. The prototype consists of a rigid body, three active tail links and a passive caudal fin. In order to analyze the guidance states of the robotic fish, 6-DoF body motion equations are presented in this paper. These equalities define the kinematic and dynamic behaviors of the robotic fish in two coordinate frames and also include the hydrodynamic forces and moments acting on the robotic fish body. The moving coordinate frame is fixed to the body and it is expressed as a body-fixed reference frame. The origin of the body-fixed frame is pointed with the center of gravity. Also, the motion of the body-fixed frame depends on the inertial reference frame that is named the earth-fixed frame. The 6-DoF motion of the robotic fish prototype in the Earth-Fixed frame with translation and rotation relations is shown in Figure 9.

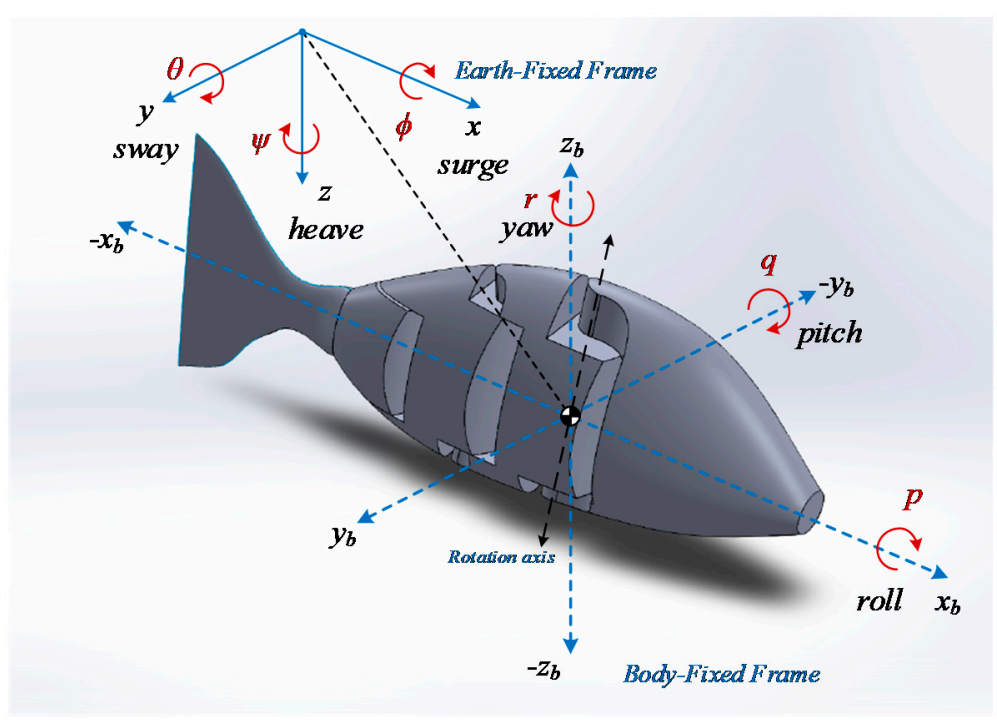

Figure 9. The designed multi-link robotic fish prototype on reference frames.

The generalized speed and positions of the robotic fish are completely given by the state variable vector $s=\left[\begin{array}{llllllllllll}u & v & w & p & q & r & x & y & z & \phi & \theta & \psi\end{array}\right]^{T}$. The linear speeds $(u, v, w)$ are surge, sway and heave, respectively. The angular speeds $(p, q, r)$ are roll, pitch and yaw speeds, respectively. Surge, sway and heave positions $(x, y, z)$ are determined with the earth-fixed frame. Roll, pitch and yaw angles $(\phi, \theta, \psi)$ are the rigid body orientations with respect to the earth-fixed frame.

The kinetic translation between the body-fixed and the earth-fixed frames for the robotic fish motion is expressed as:

$$
\dot{\eta}=\left[\begin{array}{cc}
R_{\Theta}(\eta) & 0_{3 \times 3} \\
0_{3 \times 3} & T_{\Theta}(\eta)
\end{array}\right] v
$$

where, $\eta=\left[\begin{array}{llllll}x & y & z & \phi & \theta & \psi\end{array}\right]^{T}$ and $v=\left[\begin{array}{llllll}u & v & w & p & q & r\end{array}\right]^{T}$ are the generalized positions and speeds, respectively. $\eta$ is relative with the body-fixed frame and speed vector depends on the earth-fixed frame. $R_{\Theta}(\eta)$ is the rotation matrix and $T_{\Theta}(\eta)$ is the transformation matrix. In this way, the $3 \mathrm{D}$ equation of the rigid body motion is given by:

$$
M \dot{v}+C(v) v+D(v) v+g(\eta)=\tau_{f}
$$


where, $M=M_{R B}+M_{A}$ is the mass matrix, $M_{R B}$ is the rigid body mass matrix and $M_{A}$ is the added mass matrix. $C(v)=C_{R B}(v)+C_{A}(v)$ is the Coriolis and centripetal matrix, $C_{R B}(v)$ is the rigid body Coriolis matrix and $C_{A}(v)$ is the hydrodynamic Coriolis matrix. $D(v)$ is the hydrodynamic damping matrix including linear and non-linear damping effects. $\tau_{f}$ is the vector that includes the hydrodynamic forces. Rigid body mass matrix and added mass matrix are defined as:

$$
\begin{gathered}
M_{R B}=\left[\begin{array}{ccccc}
m I_{3 \times 3} & -m S\left(r_{G}\right) \\
m S\left(r_{G}\right) & I_{0}
\end{array}\right] \\
M_{A}=\left[\begin{array}{ccccccc}
X_{\dot{u}} & 0 & 0 & 0 & 0 & 0 \\
0 & Y_{\dot{v}} & 0 & 0 & 0 & Y_{\dot{r}} \\
0 & 0 & Z_{\dot{w}} & 0 & Z_{\dot{q}} & 0 \\
0 & 0 & 0 & K_{\dot{p}} & 0 & 0 \\
0 & 0 & M_{\dot{w}} & 0 & M_{\dot{q}} & 0 \\
0 & N_{\dot{v}} & 0 & 0 & 0 & N_{\dot{r}}
\end{array}\right]
\end{gathered}
$$

In these equations, $m$ is the total body mass. The position of the center of gravity is equal to $r_{G}=\left[\begin{array}{ccc}x_{G} & y_{G} & z_{G}\end{array}\right]^{T}$. The moments of inertia are defined as $I_{0}$. Asymmetric matrix $S$ is used for easier computation. $X_{\dot{u}}$ and so forth are the zero-frequency added mass coefficients. $C_{R B}(v)$ matrix indicates the Coriolis matrix $\left[\begin{array}{lll}p & q & r\end{array}\right]^{T} \times\left[\begin{array}{lll}u & v & w\end{array}\right]^{T}$ and it is expressed by:

$$
C_{R B}(v)=\left[\begin{array}{cc}
0_{3 \times 3} & -S\left(v_{1} m I_{3 \times 3}-v_{2} m S\left(r_{G}\right)\right) \\
-S\left(v_{1} m I_{3 \times 3}+v_{2}-m S\left(r_{G}\right)\right) & -S\left(v_{1} m S\left(r_{G}\right)+v_{2} I_{0}\right)
\end{array}\right]
$$

$C_{A}(v)$ matrix contains Munk moments and it is given by:

$$
C_{A}(v)=\left[\begin{array}{cccccc}
0 & 0 & 0 & 0 & -Z_{\dot{w}} w & Y_{\dot{v}} v \\
0 & 0 & 0 & Z_{\dot{w}} w & 0 & -X_{\dot{u}} u \\
0 & 0 & 0 & -Y_{\dot{v}} v & X_{\dot{u}} u & 0 \\
0 & -Z_{\dot{w}} w & Y_{\dot{v}} v & 0 & -N_{\dot{r}} r & M_{\dot{q}} q \\
Z_{\dot{w}} w & 0 & -X_{\dot{u}} u & N_{\dot{r}} r & 0 & -K_{\dot{p}} p \\
-Y_{\dot{v}} v & X_{\dot{u}} u & 0 & -M_{\dot{q}} q & K_{\dot{p}} p & 0
\end{array}\right]
$$

Hydrodynamic damping effects of the robotic fish are separated into linear and non-linear terms. These matrixes can be defined as below equations:

$$
\begin{aligned}
& D_{L}=\left[\begin{array}{cccccc}
X_{u} & 0 & 0 & 0 & 0 & 0 \\
0 & Y_{v} & 0 & 0 & 0 & -Y_{r} \\
0 & 0 & Z_{w} & 0 & Z_{q} & 0 \\
0 & 0 & 0 & K_{p} & 0 & 0 \\
0 & 0 & M_{w} & 0 & M_{q} & 0 \\
0 & -N_{v} & 0 & 0 & 0 & N_{r}
\end{array}\right] \\
& D_{N L}=\left[\begin{array}{cccccc}
X_{|u| u}|u| & 0 & 0 & 0 & 0 & 0 \\
0 & Y_{|v| v \mid}|v| & 0 & 0 & 0 & -Y_{|v| r}|v| \\
0 & 0 & Z_{|w| w \mid}|w| & 0 & Y_{|w| q \mid}|w| & 0 \\
0 & 0 & 0 & K_{|p| p \mid}|p| & 0 & 0 \\
0 & 0 & M_{|q| w \mid}|q| & 0 & M_{|q| q}|q| & 0 \\
0 & -N_{|r| v \mid}|r| & 0 & 0 & 0 & N_{|r| r \mid}|r|
\end{array}\right]
\end{aligned}
$$


$D_{L}$ and $D_{N L}$ are the diagonal matrixes and these matrixes depend on the body shape and speed of the robotic fish, respectively. $X_{u}, X_{|u| u}$ and so forth terms are linear and non-linear positive damping coefficients. In marine applications, underwater vehicles move at low speed and adding mass terms can be neglected. However, these effects are used to obtain complete motion model.

$W=m g$ is the gravity force and $B=\rho g \Delta$ is the buoyancy force. Here, $\Delta$ is the submerged volume. Thus, hydrostatic effect $g(\eta)$ is calculated as:

$$
g(\eta)=\left[\begin{array}{c}
(W-B) s(\theta) \\
-(W-B) c(\theta) s(\phi) \\
-(W-B) c(\theta) c(\phi) \\
-\left(y_{g} W-y_{b} B\right) c(\theta) c(\phi)+\left(z_{g} W-z_{b} B\right) c(\theta) s(\phi) \\
-\left(z_{g} W-z_{b} B\right) s(\theta)+\left(x_{g} W-x_{b} B\right) c(\theta) c(\phi) \\
-\left(x_{g} W-x_{b} B\right) c(\theta) s(\phi)-\left(y_{g} W-y_{b} B\right) s(\theta)
\end{array}\right]
$$

Note that $(s(), c(), t())$ are equal to $(\sin (), \cos (), \tan ()) \cdot r_{B}=\left[\begin{array}{lll}x_{B} & y_{B} & z_{B}\end{array}\right]^{T}$ is the position vector from the body origin to center of buoyancy. The force vector $\tau_{f}$ includes the hydrodynamic forces and moments acting on the fish body and it is expressed as:

$$
\tau_{f}=\left[\begin{array}{cccccc}
F_{x} & F_{y} & F_{z} & \tau_{x} & \tau_{y} & \tau_{z}
\end{array}\right]^{T}
$$

where, $\left(F_{x}, F_{y}, F_{z}\right)$ represent the surge, sway and heave forces. $\left(\tau_{x}, \tau_{y}, \tau_{z}\right)$ are the yaw, pitch and roll moments, respectively. It is considered that $F_{x}=F_{F} \cos \left(\theta_{2}+\theta_{3}+\theta_{4}\right) . F_{y}$ and $F_{z}$ are chosen as zero, and $\tau_{z}=\left(\left(l_{1}+l_{2}\right) \cos \left(\theta_{2}\right)+l_{3} \cos \left(\theta_{2}+\theta_{3}\right)+l_{4} \cos \left(\theta_{2}+\theta_{3}+\theta_{4}\right)\right) F_{F} \sin \left(\theta_{2}+\theta_{3}+\theta\right)$. Finally, 6-DoF motion block diagram is given in Figure 10 that includes the fish body and fish tail equations both body-fixed frame and earth-fixed frame.

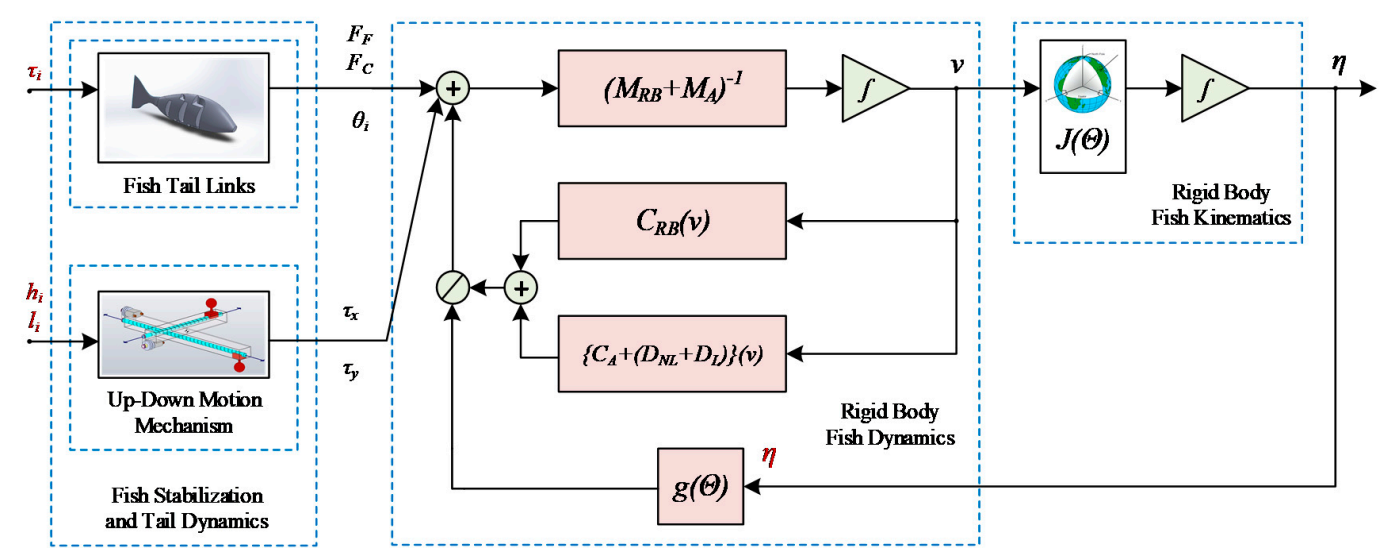

Figure 10. 6-DoF motion block diagram.

By solving these equations, whole 3D non-linear dynamic model of the robotic fish is obtained.

\section{Implementations of the Fish-Like Motion}

In order to mimic real fish swimming abilities, the robotic fish model focuses on five special points according to the real carp:

1. Proportional optimum link lengths according to actual size,

2. Flapping frequency,

3. Swimming speed performance,

4. Proportional physical parameters according to the carp,

5. Trajectory tracking. 
As can be observed from the experiments, proportional link lengths generate sinusoidal angles while the robotic fish propels itself. This situation causes the sinusoidal undulation motions. Secondly, obtained flapping frequency from the observations is an appropriate value and it is applied to the robotic fish model. Forward and turning speeds of the carp are also examined in the simulations. Finally, guidance trajectory tracking is performed to validate the dynamic model.

\subsection{Ability of the Fish-Like Motion}

In this study, numerical analyses are performed in a MATLAB environment. The flapping frequency of the carp is observed from the experiments and it is determined as $2.857 \mathrm{~Hz}$. This value is also applied to the robotic fish model to validate the ability of the fish-like motion. In Figure 11, free-swimming motion of the robotic fish is illustrated during an $80 \mathrm{~s}$ simulation time.

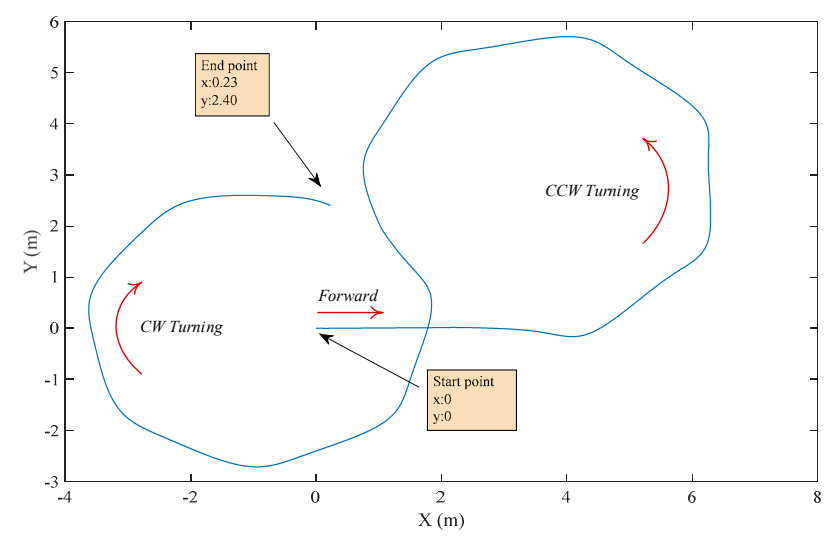

Figure 11. Free-swimming motion of the robotic fish. It is noted that there are three swimming modes: Forward, Counter Clock Wise (CCW) and Clock Wise (CW) turning.

Figure 11 shows open loop free-swimming behavior on a two-dimensional (2D) reference frame. The start position of the robotic fish is $X=0 \mathrm{~m}, \mathrm{Y}=0 \mathrm{~m}$. After $80 \mathrm{~s}$ simulation time, the robotic fish reaches the final position at $X=0.23 \mathrm{~m}, \mathrm{Y}=2.40 \mathrm{~m}$. The speed of the robotic fish is measured as $0.184 \mathrm{~m} / \mathrm{s}$ and $0.474 \mathrm{~m} / \mathrm{s}$ for forward and turning swimming motions, respectively. Figure 12 shows the link angles and Figure 13 presents the thrust force on the $x$-axis.

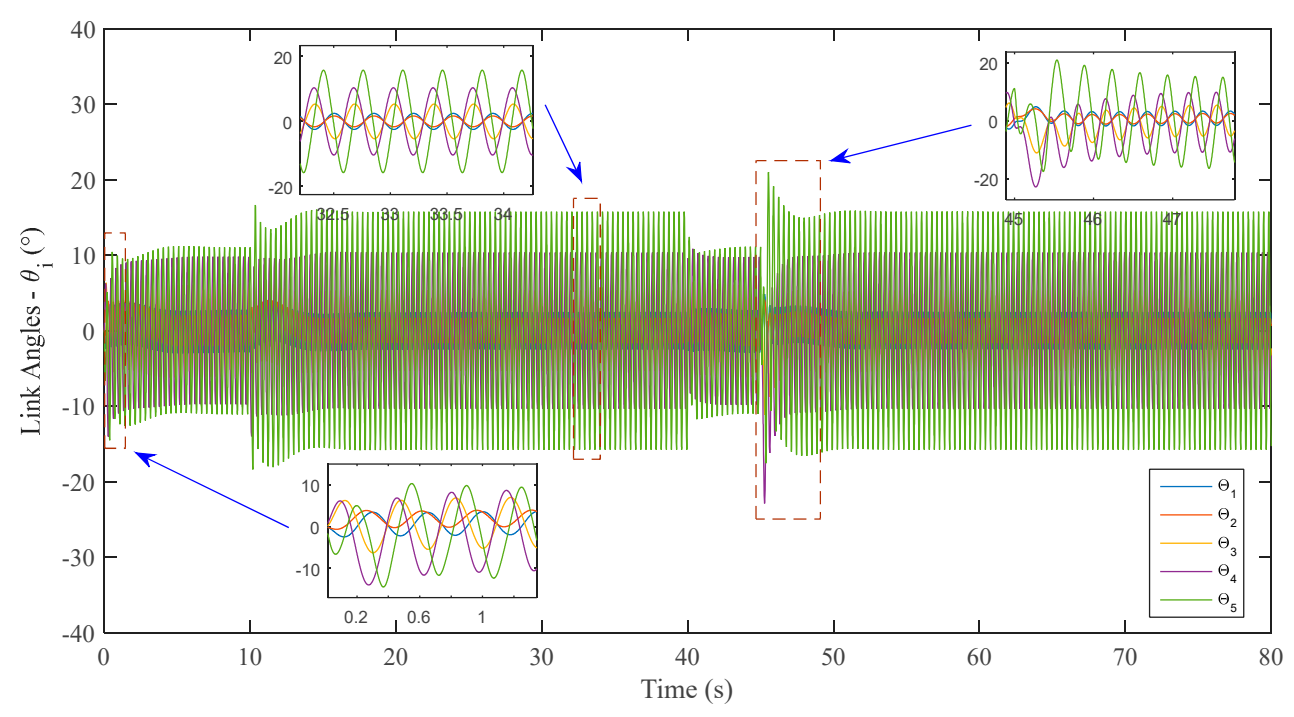

Figure 12. Link angles of the robotic fish for free-swimming motion during $80 \mathrm{~s}$ simulation time. 


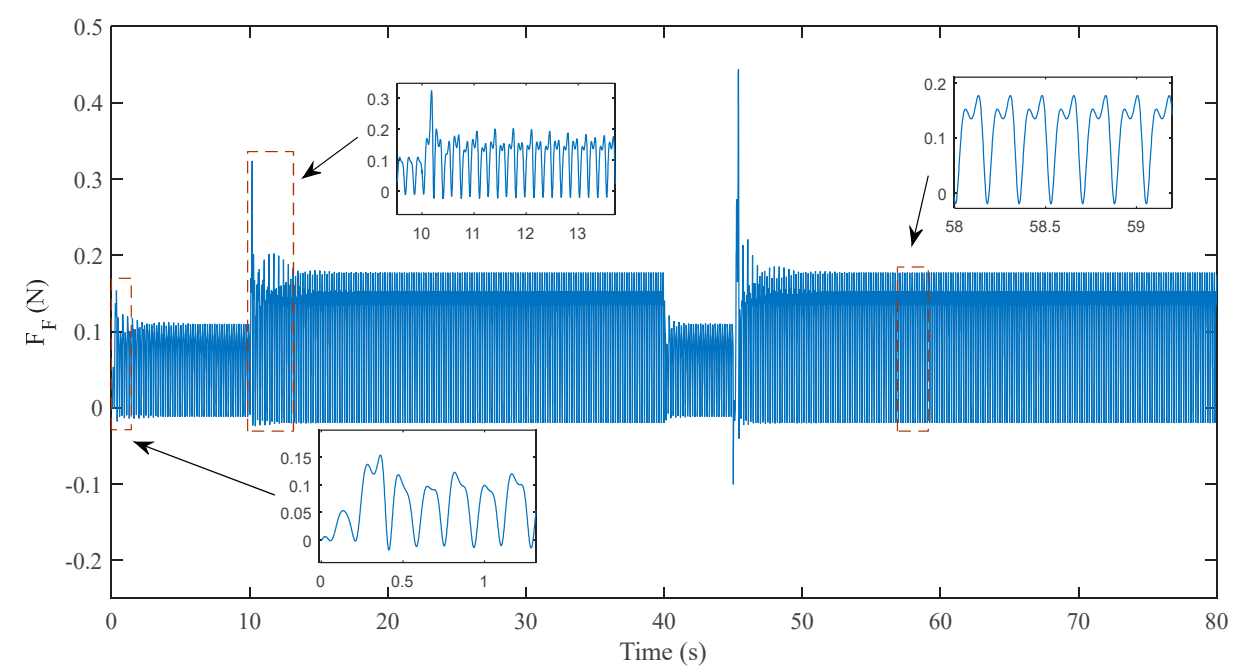

Figure 13. Thrust force of the robotic fish for free-swimming motion during 80 s simulation time.

In Figure 12, tail link behaviors are illustrated for different swimming motions. The robotic fish tail swings itself with sinusoidal undulations with pure sine waves.

The thrust force of the tail is determined as nearly $0.110 \mathrm{~N}$ and $0.177 \mathrm{~N}$ for forward and turning swimming modes.

\subsection{Guidance and Trajectory Tracking}

The proposed model of the robotic fish including guidance and trajectory tracking consists of a three-layered hierarchical architecture: inner loop, outer loop and trajectory generator. The inner loop is controlled with the simple and practical control structure inspired by Reaching Law Control (RLC) approach. The inner loop occurs in the body frame of the robotic fish. The outer loop deals with the guidance control structure. On the other hand, the trajectory generator builds up the 3D path tracking in earth-fixed frame. The block diagram of the closed loop system is shown in Figure 14.

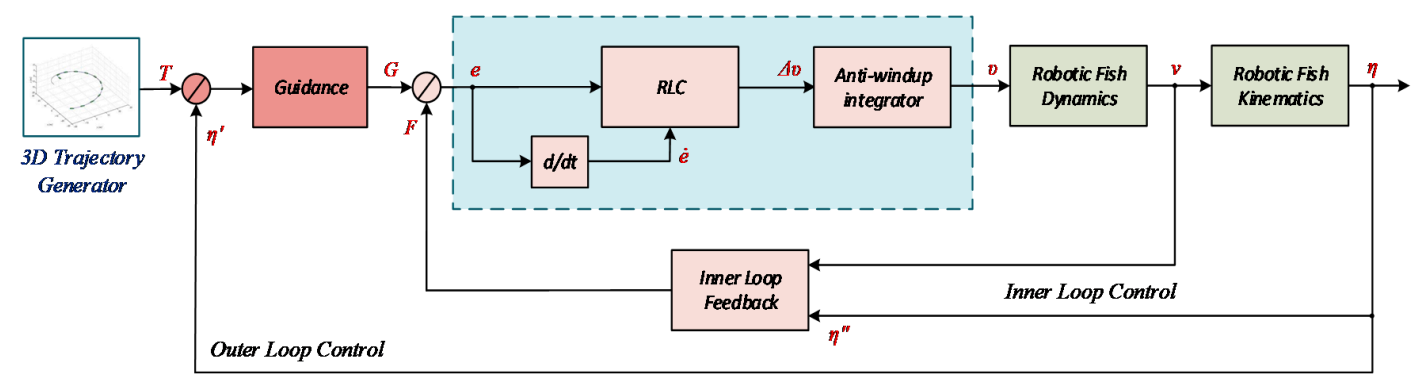

Figure 14. Block diagram of the proposed guidance and trajectory tracking with RLC.

In Figure $14, T, \eta, G$ and $F$ indicate reference trajectory, current positions, reference of the guidance, and current orientations and speed, respectively. These parameters are defined as below:

$$
\left\{\begin{array}{l}
T=\left[\begin{array}{lll}
X_{\text {ref }} & Y_{\text {ref }} & Z_{\text {ref }}
\end{array}\right]^{T} \\
\eta^{\prime}=\left[\begin{array}{lll}
x & y & z
\end{array}\right]^{T} \\
\eta^{\prime \prime}=\left[\begin{array}{lll}
\phi & \theta & \psi
\end{array}\right]^{T} \\
G=\left[\begin{array}{lll}
\theta_{\text {ref }} & \psi_{\text {ref }} & v_{\text {ref }}
\end{array}\right]^{T} \\
F=\left[\begin{array}{lll}
\theta & \psi & v
\end{array}\right]^{T}
\end{array}\right.
$$


It is noted that the reference matrix of the guidance is calculated by using a trigonometrical approach and that each element of the matrix can be given by Equation (23):

$$
\left\{\begin{array}{l}
\theta_{r e f}=-\tan ^{-1}\left(\left(z_{r e f}-z\right) /\left(\sqrt{\left(x_{r e f}-x\right)^{2}+\left(y_{r e f}-y\right)^{2}}\right)\right) \\
\psi_{r e f}=\tan ^{-1}\left(\left(y_{r e f}-y\right) /\left(x_{r e f}-x\right)\right) \\
v_{r e f}=\zeta \sqrt{\left(x_{r e f}-x\right)^{2}+\left(y_{r e f}-y\right)^{2}+\left(z_{r e f}-z\right)^{2}}
\end{array}\right.
$$

here, $\zeta$ is a constant speed control gain. In the general RLC approach, the discrete time reaching law [36,37] can be given by:

$$
\Delta S(k+1)=-\sigma \operatorname{sgn}(S(k))-\alpha S(k)
$$

where $\sigma$ and $\alpha$ are constant parameters. $\Delta S(k+1)$ is then expressed by:

$$
\Delta S(k+1)=\frac{S(k+1)-S(k)}{T_{S}}
$$

$T_{S}$ is the sampling time. With the assumption of $\{\Delta S(0)=0\}$, discrete time switching function is given as:

$$
S(k)=\lambda e(k)+\Delta e(k)
$$

here, $e(k)$ is the error of the guidance and $\Delta e(k)$ is the derivative of the error in the $k$ th sampling step. $\lambda$ is the slope of the switching line and indicates a first-order dynamics of the error. The high-frequency oscillation problem known as chattering is occurred if Equation (24) is used directly in practice [37,38]. This chattering problem is presented in Figure 15 and it can be solved by using sat $(S(k))$ function instead of $\operatorname{sgn}(S(k))$. sat $(\cdot)$ function is expressed by:

$$
\operatorname{sat}(\Omega)=\left\{\begin{array}{cc}
\Omega & \text { if }|\Omega| \leq 1 \\
\operatorname{sgn}(\Omega) & \text { if }|\Omega|>1
\end{array}\right.
$$

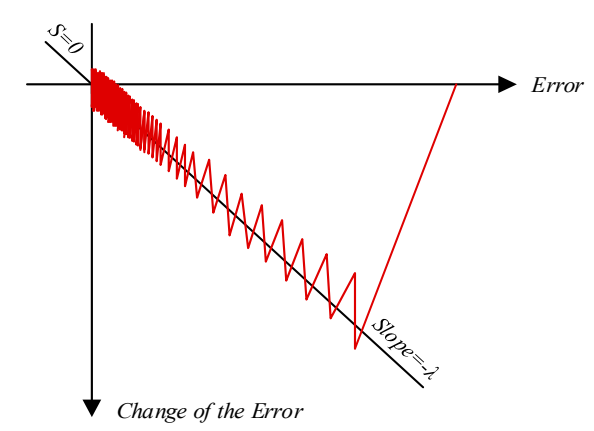

Figure 15. A typical high-frequency oscillation problem.

The new reaching law can be obtained as:

$$
\Delta S(k+1)=-\sigma s a t(S(k))-\alpha S(k)
$$

The reaching law is also rewritten as below with this sat $(\cdot)$ function in the Boundary Layer (BL):

$$
\Delta S(k+1)=-\beta S(k)
$$

where $\beta=(\sigma / B L)+\alpha$. Thus, the control output becomes:

$$
\Delta v(k)=K S(k)
$$


Since $S$ is forced to reach zero with this RLC approach, exponentially reducing of the error to zero with time constant of $(1 / \lambda)$ is provided.

Also, an anti-windup integrator is added to stop over-integration for the protection of the designed model as seen in Figure 16.

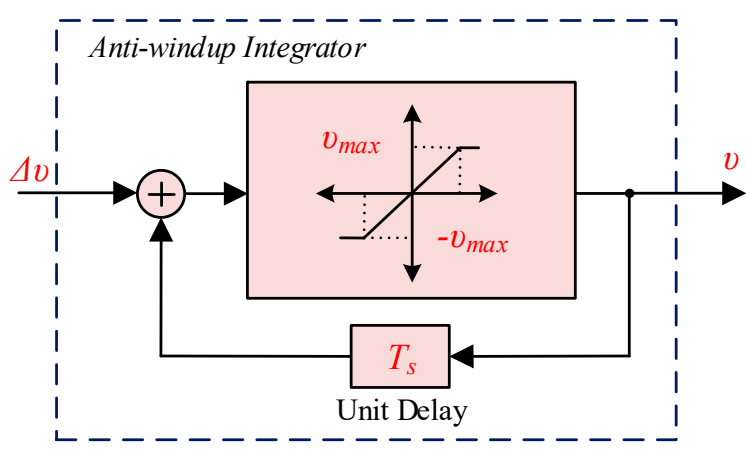

Figure 16. Thrust force of the robotic fish for free-swimming motion during $80 \mathrm{~s}$.

6-DoF motion responses completely include the generalized linear and angular positions and speeds of the robotic fish. Positions (X, Y, Z) are determined with the earth-fixed frame. Roll, pitch and yaw angles $(\phi, \theta, \psi)$ are also rigid body orientations with respect to the earth-fixed frame. In order to validate the accuracy of the designed model, simulations are examined in this section.

Figure 17a shows the closed loop forward speed responses of the robotic fish from $0.1 \mathrm{~m} / \mathrm{s}$ to $0.4 \mathrm{~m} / \mathrm{s}$. These speed values are chosen as observed speed ranges of the carp. Figure $17 \mathrm{~b}$ shows the orientation control that includes yaw angles of the robotic fish model during $40 \mathrm{~s}$ simulation time. The control performance of the closed loop system is summarized in Table 1.

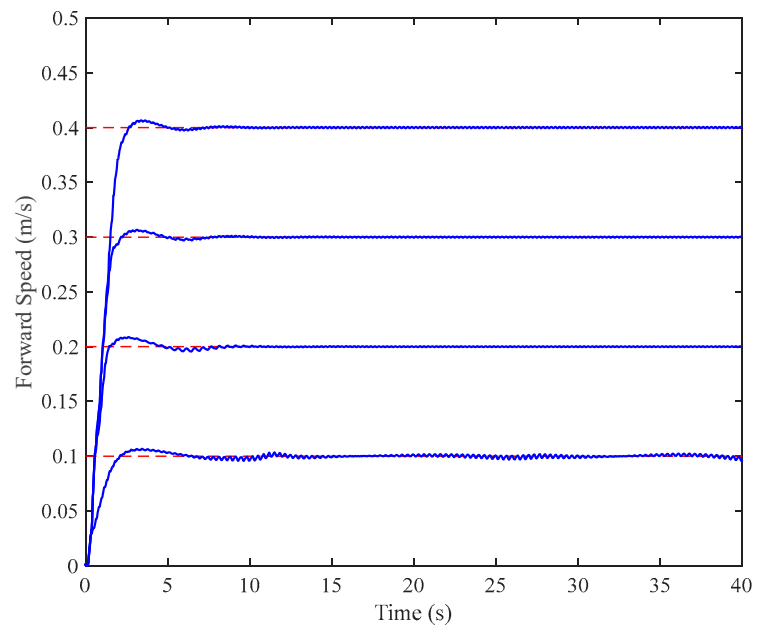

(a)

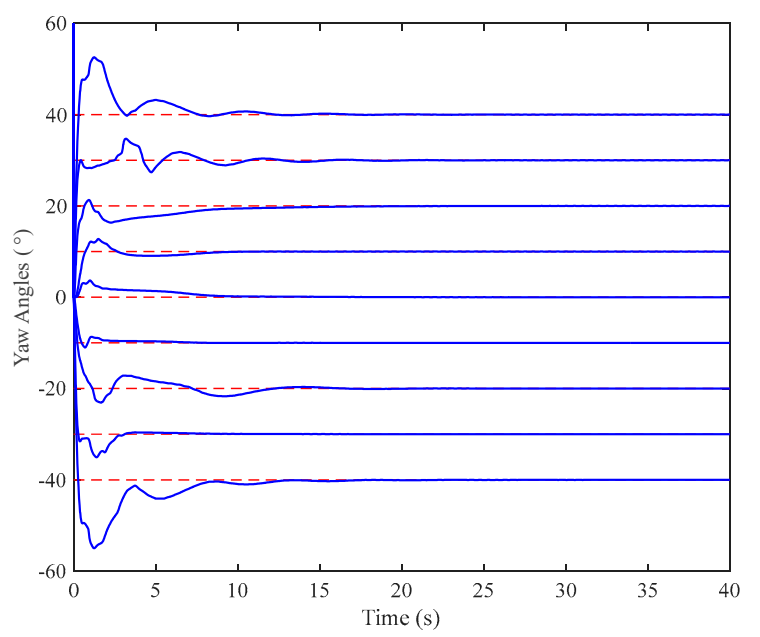

(b)

Figure 17. Closed loop responses of the 6-DoF system during 40 s simulation time: (a) Forward speed control; (b) Yaw angles tracking control.

Another key point is to realize pitch and roll motions together. In Figure 18, these motions are performed for step and stairs references. The closed loop control performance of the roll and pitch motions are illustrated in Table 2.

In order to validate the closed loop system, two different path trajectories are also generated in earth-fixed frame for 3D trajectory tracking. First path is a $3 \mathrm{D}$ sinusoidal path $\left(\mathrm{P}_{1}\right)$ reference and the second path is a 3D CCW circular path $\left(\mathrm{P}_{2}\right)$ reference. Trajectory tracking responses indicate the 
time-dependent performances of the robotic fish model. Figure 19 shows the closed loop $\mathrm{P}_{1}$ tracking performance during a $60 \mathrm{~s}$ simulation time.

Amplitude and frequency of the $\mathrm{P}_{1}$ reference are $\pm 0.18 \mathrm{~m}$ and $0.2 \mathrm{~Hz}$, respectively. The depth of $P_{1}$ is $3 \mathrm{~m}$. The robotic fish tracks the $P_{1}$ reference with a speed of $0.42 \mathrm{~m} / \mathrm{s}$. Figure 20 presents the $P_{1}$ tracking errors of speed and orientation angles.

Table 1. The closed loop control performance: Maximum overshoot (\%) and settling time (s) of forward speed for $(0.1 \mathrm{~m} / \mathrm{s})-(0.4 \mathrm{~m} / \mathrm{s})$ and yaw angles for $\left(-40^{\circ}\right)-\left(+40^{\circ}\right)$.

\begin{tabular}{cccc}
\hline & Interval & Maximum Overshoot (\%) & Settling Time (s) \\
\hline \multirow{3}{*}{ Forward Speed } & 0.1 & 6.80 & 16.30 \\
$(\mathrm{~m} / \mathrm{s})$ & 0.2 & 4.40 & 11.80 \\
& 0.3 & 2.23 & 15.40 \\
& 0.4 & 1.65 & 12.20 \\
\hline & -40 & 37.50 & 18.30 \\
& -30 & 16.66 & 8.80 \\
Yaw Angles $\left(^{\circ}\right)$ & -20 & 15 & 16.65 \\
& -10 & 10 & 8.20 \\
& 0 & 36 & 14.50 \\
& 10 & 27.50 & 10.80 \\
& 20 & 6.50 & 21.50 \\
& 30 & 15 & 17.60 \\
& 40 & 31.25 & 18.90 \\
\hline
\end{tabular}

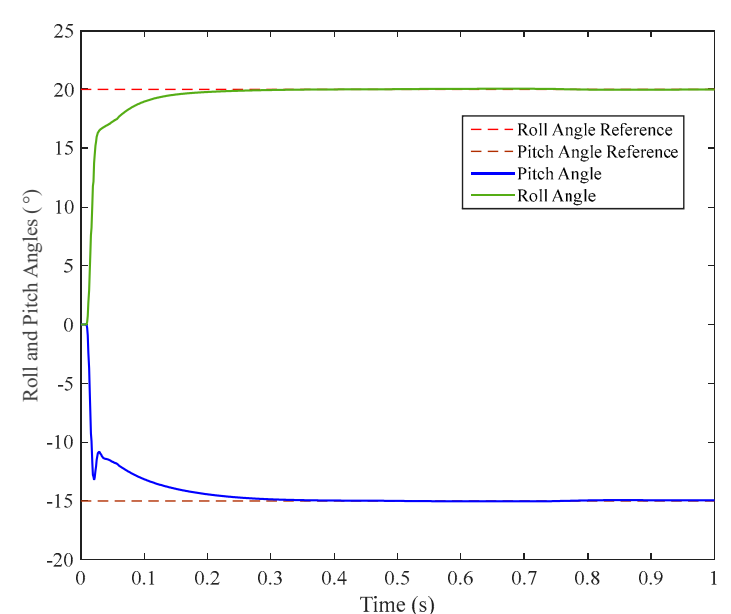

(a)
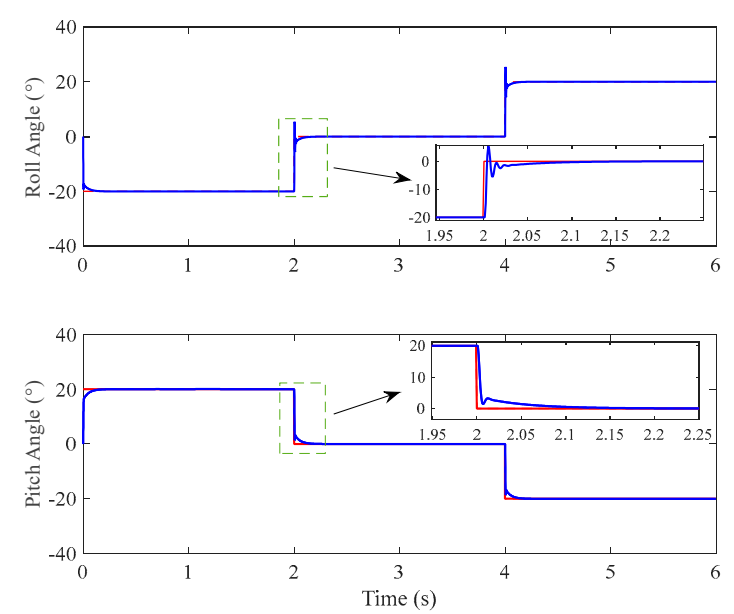

(b)

Figure 18. Roll and pitch motions together: (a) Step response; (b) Stairs response.

Table 2. The simultaneously closed loop control performance of the roll and pitch motions for step and stairs references.

\begin{tabular}{|c|c|c|c|c|c|c|c|c|}
\hline \multirow{3}{*}{$\begin{array}{l}\text { Criteria } \\
\text { Value }\left(^{\circ}\right) \\
\text { Motion }\end{array}$} & \multicolumn{2}{|c|}{ Step } & \multicolumn{6}{|c|}{ Stairs } \\
\hline & \multirow{2}{*}{$\begin{array}{c}20 \\
\text { Roll }\end{array}$} & \multirow{2}{*}{$\begin{array}{l}-15 \\
\text { Pitch }\end{array}$} & \multicolumn{2}{|c|}{-20} & \multicolumn{2}{|c|}{0} & \multicolumn{2}{|c|}{20} \\
\hline & & & Roll & Pitch & Roll & Pitch & Roll & Pitch \\
\hline $\begin{array}{c}\text { Maximum } \\
\text { Overshoot (\%) }\end{array}$ & - & - & - & - & 27 & - & 27.50 & - \\
\hline Settling Time (s) & 0.38 & 0.37 & 0.20 & 0.24 & 0.18 & 0.26 & 0.19 & 0.24 \\
\hline
\end{tabular}




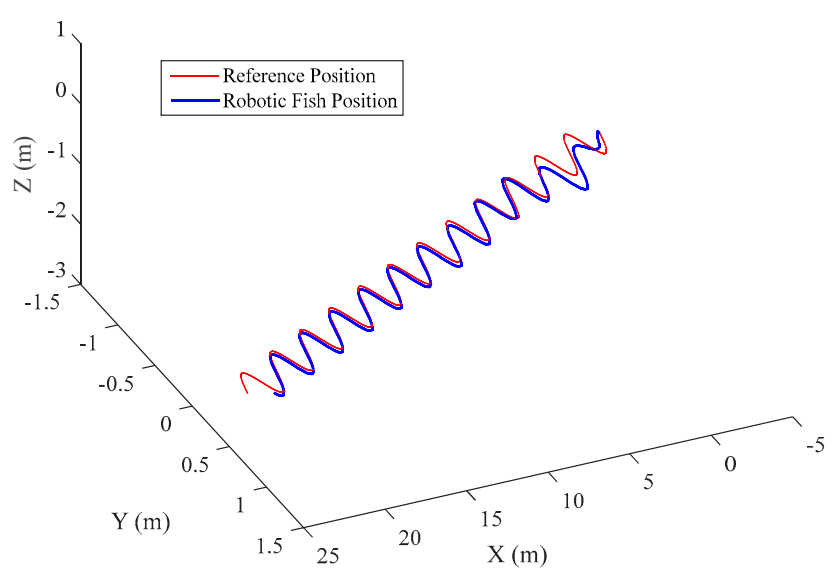

Figure 19. $3 \mathrm{D} \mathrm{P}_{1}$ tracking control performance.
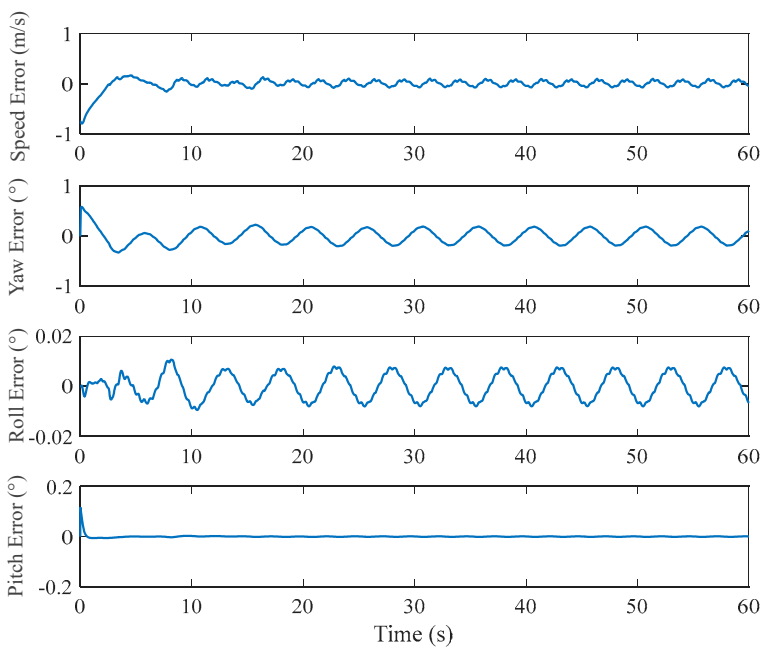

Figure 20. Errors for $\mathrm{P}_{1}$ tracking.

6-DoF motion responses including positions, yaw, roll, pitch and also link angles are given in Figure 21. The robotic fish swings the flexible tail to keep the $\mathrm{P}_{1}$ reference during closed loop tracking.
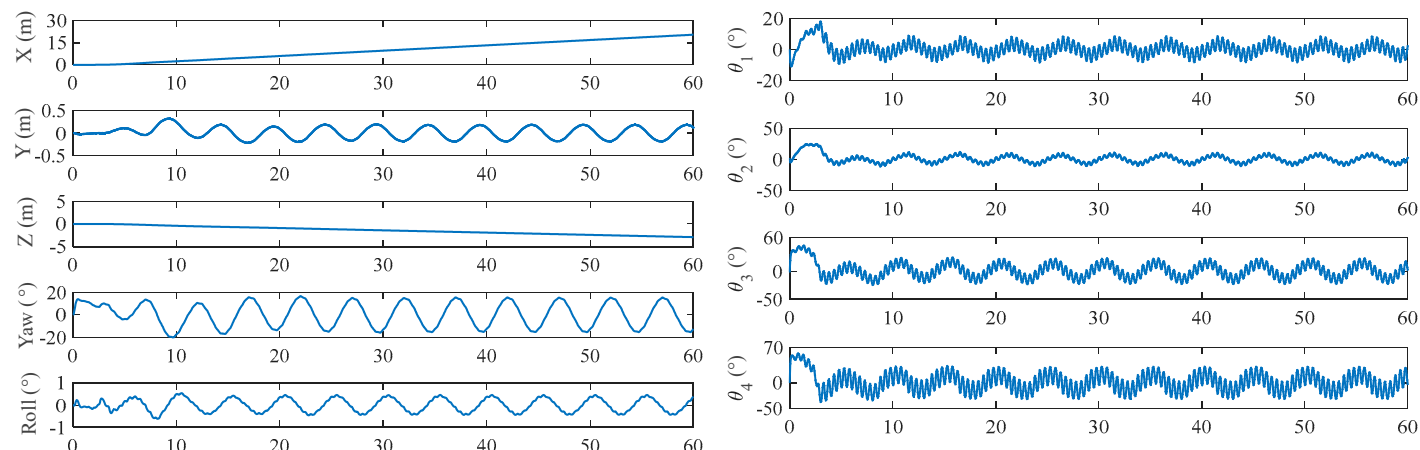

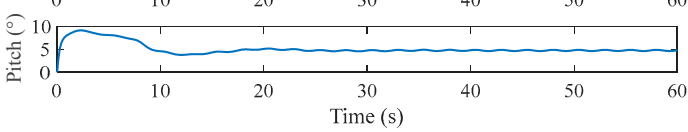

(a)
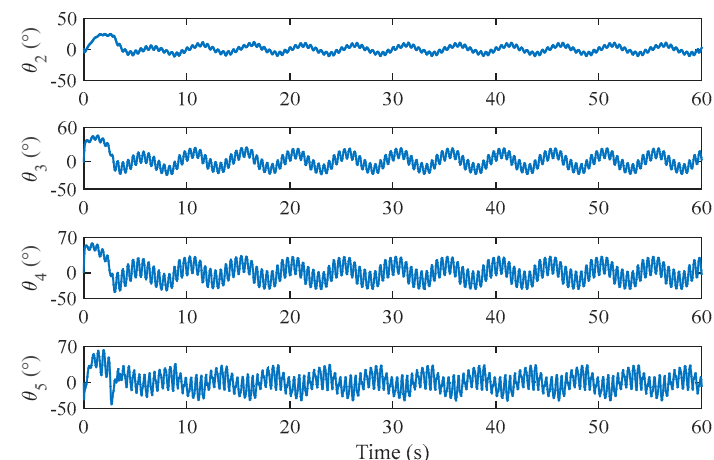

(b)

Figure 21. $\mathrm{P}_{1}$ tracking responses: (a) 6-DoF responses; (b) Link angles. 
Figure 22 shows the closed loop $\mathrm{P}_{2}$ tracking performance during a $64 \mathrm{~s}$ simulation time. Diameter of the $\mathrm{P}_{2}$ is $8 \mathrm{~m}$ and depth is $1.28 \mathrm{~m}$. Initial positions of the robotic fish and $\mathrm{P}_{2}$ reference begin the origin at $\mathrm{X}=0 \mathrm{~m}, \mathrm{Y}=0 \mathrm{~m}, \mathrm{Z}=0 \mathrm{~m}$.

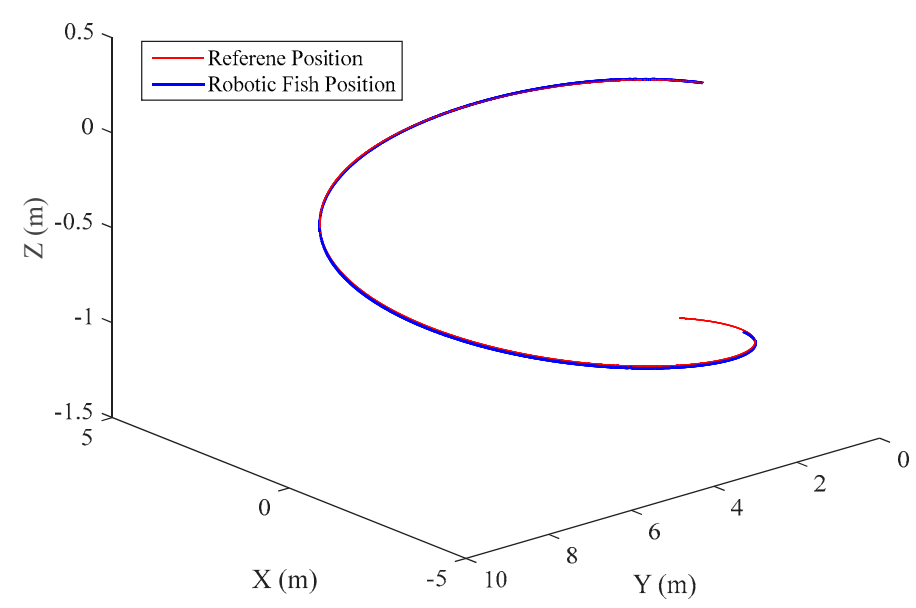

Figure 22. $3 \mathrm{D} \mathrm{P}_{2}$ tracking control performance.

The robotic fish turns around the CCW direction to track the $\mathrm{P}_{2}$ reference with a speed of $0.4 \mathrm{~m} / \mathrm{s}$. Figure 23 also presents the $\mathrm{P}_{2}$ tracking errors of speed and orientation angles.
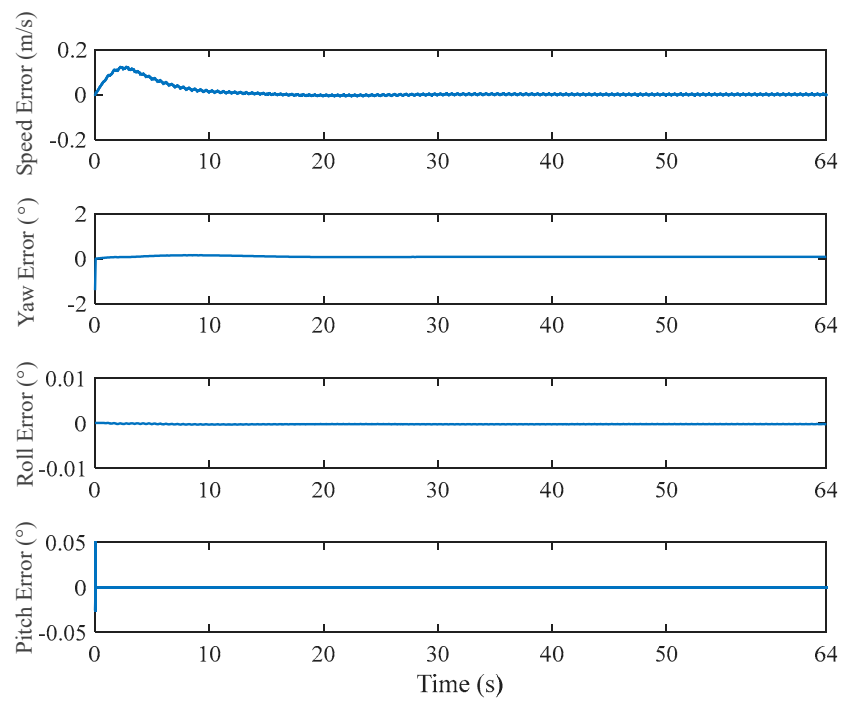

Figure 23. Errors for $\mathrm{P}_{2}$ tracking.

6-DoF motion responses are given in Figure 24. When the robotic fish reaches the steady state, link angles become stable and generate small amplitudes.

As shown in Table 3, the tracking errors of $\mathrm{P}_{1}$ and $\mathrm{P}_{2}$ references on the $\mathrm{x}, \mathrm{y}$ and $\mathrm{z}$ axis are evaluated by Root Mean Square Error (RMSE) performance index and given as:

$$
R M S E=\sqrt{\frac{\sum_{k=1}^{n} e(k)^{2}}{n}}
$$




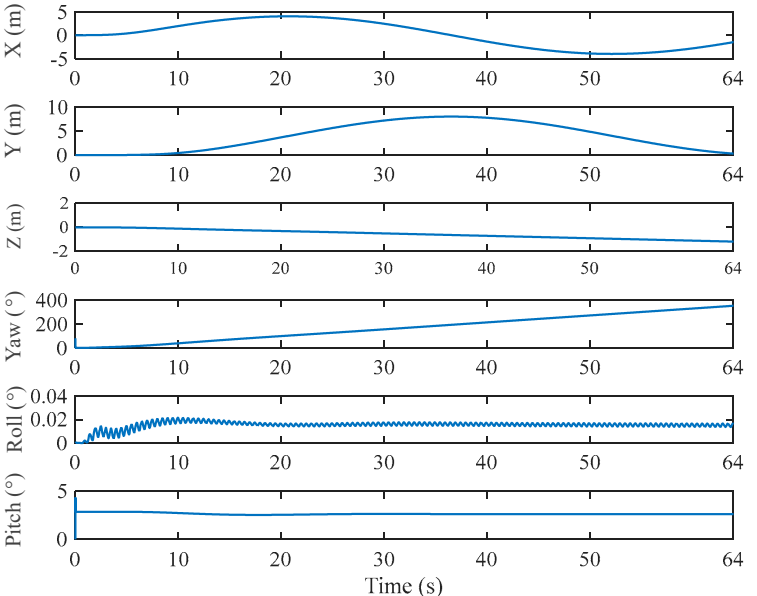

(a)

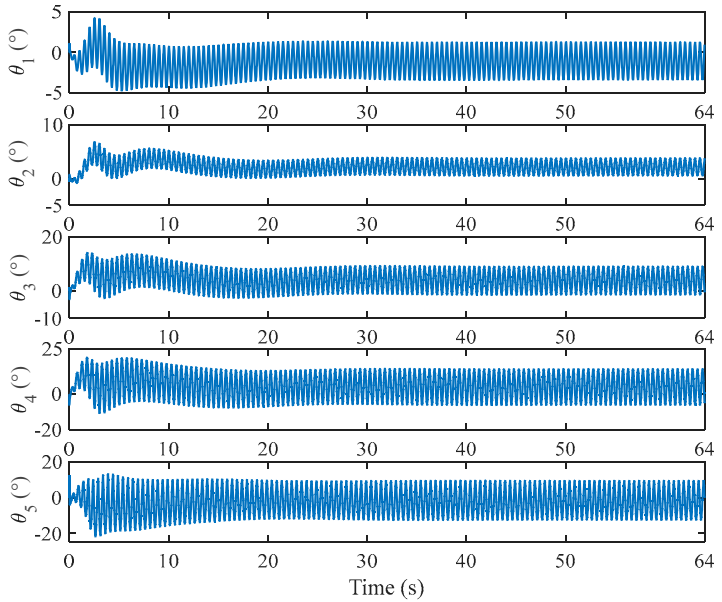

(b)

Figure 24. $\mathrm{P}_{2}$ tracking responses: (a) 6-DOF responses; (b) Link angles.

Table 3. The tracking errors for proposed closed loop control system: $\mathrm{P}_{1}$ and $\mathrm{P}_{2}$ indicate the generated 3D path trajectories.

\begin{tabular}{cccc}
\hline Trajectory & $e_{x}(\mathrm{~m})$ & $e_{y}(\mathrm{~m})$ & $e_{z}(\mathrm{~m})$ \\
\hline $\mathrm{P}_{1}$ & 0.0968 & 0.0131 & 0.1058 \\
\hline $\mathrm{P}_{2}$ & 0.0827 & 0.0107 & 0.0920 \\
\hline
\end{tabular}

In order to visualize the performance of the proposed closed loop control system in a virtual reality platform, the designed robotic fish model is also adopted to Virtual Reality Modeling Language (VRML). A simulation scenario is constructed consisting of a marine environment. The goal is to perform the $\mathrm{P}_{2}$ tracking underwater. Figure 25 shows the top view above the sea, and the isometric view of the underwater environment is also shown in Figure 26.
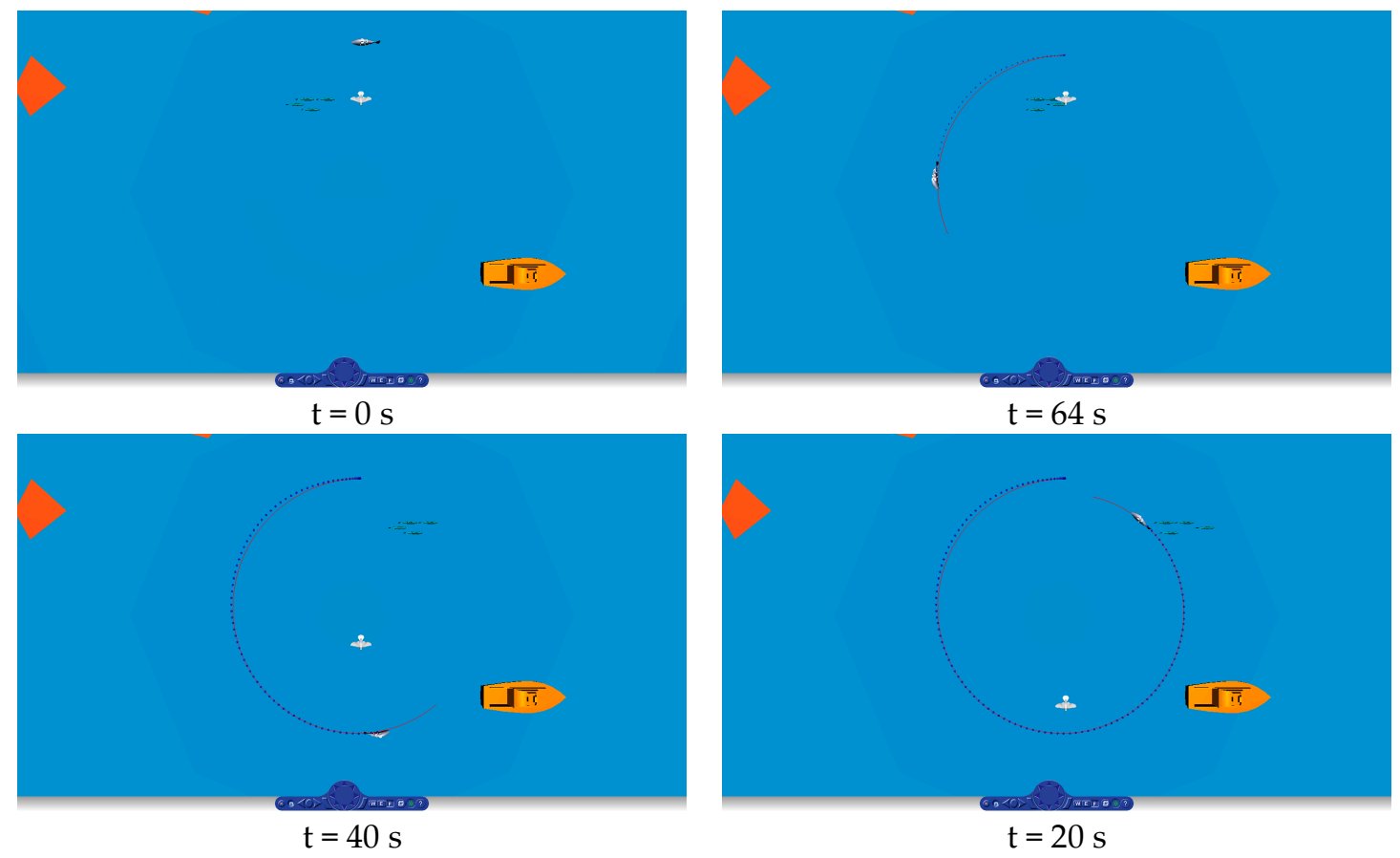

Figure 25. Top view above the sea for closed loop $\mathrm{P}_{2}$ tracking simulation. 


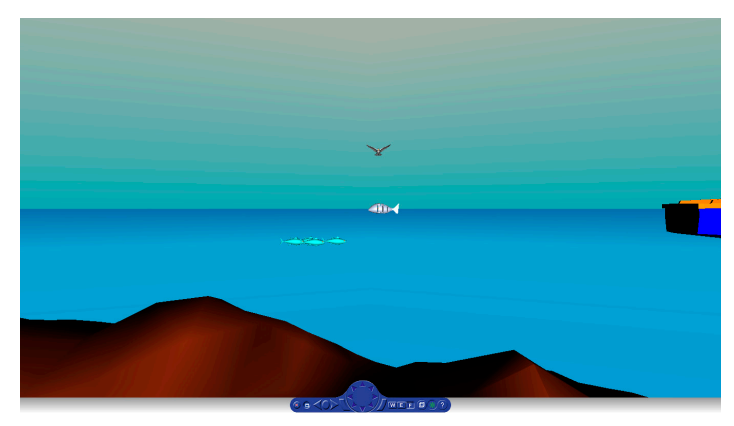

$\mathrm{t}=0 \mathrm{~s}$

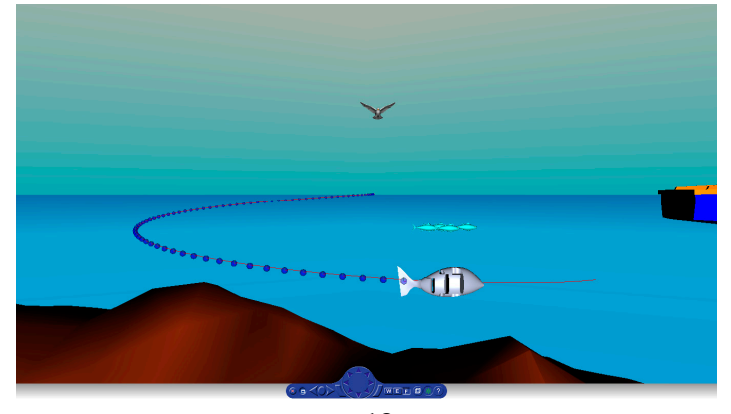

$\mathrm{t}=40 \mathrm{~s}$

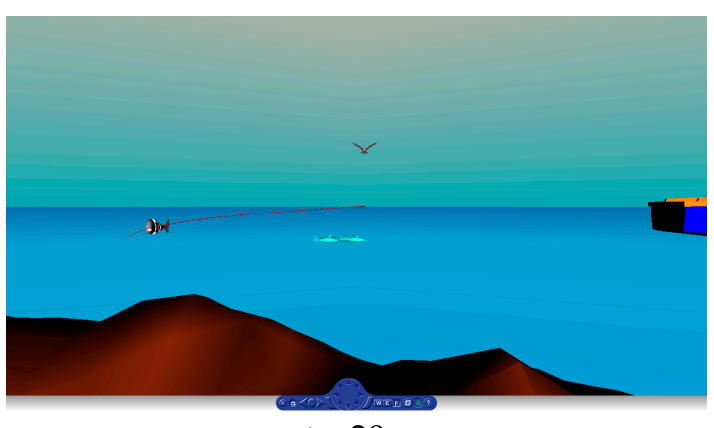

$\mathrm{t}=20 \mathrm{~s}$

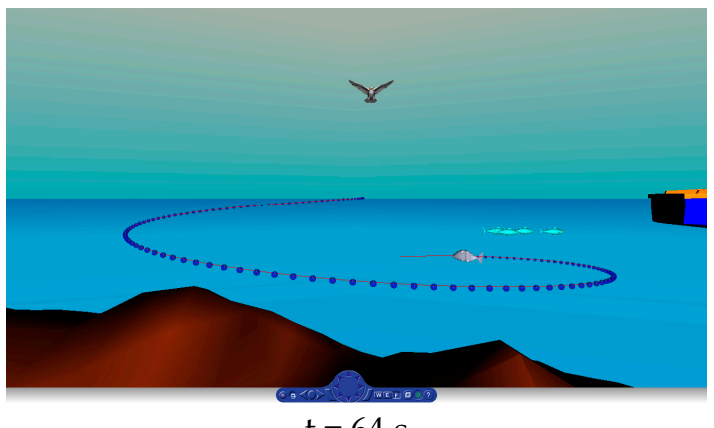

$\mathrm{t}=64 \mathrm{~s}$

Figure 26. Isometric view of the underwater environment for closed loop $\mathrm{P}_{2}$ tracking.

All of the simulation results show that the bio-inspired non-linear dynamic model of the robotic fish is suitable to mimic biological features of Carangiform carp. In this way, biomimetic modeling can be easily implemented to the robotic fish prototype in the future work.

\section{Conclusions}

This study presents a complete 3D non-linear dynamic model of a biomimetic robotic fish based on real carp locomotion. The designed model is derived by using the Lagrange formulation approach and includes both kinematic and hydrodynamic effects acting on each link. In order to achieve six degrees of freedom motions in the earth fixed frame, an up-down mechanism is designed that controls the influence of pitch and roll. This mechanism gives the position and orientation of the robotic fish in 3D space. Moreover, linear and rotational behavior responses are obtained and controlled in the simulations. In order to mimic biological features of fish with the dynamic model, various swimming patterns of a real carp are observed in the experimental pool. Link length ratio, flapping frequency, speed range, displacement and Euler angles are analyzed to determine appropriate desired inputs for the designed model. According to optimized real carp values, link lengths of the anterior body, active links and passive caudal fins are determined as $0.044 \mathrm{~m},[0.043 \mathrm{~m} 0.040 \mathrm{~m} 0.010 \mathrm{~m}]$ and $0.063 \mathrm{~m}$, respectively. Flapping frequency of carp is observed from $1.818 \mathrm{~Hz}$ to $2.857 \mathrm{~Hz}$ and it is also chosen as $2.857 \mathrm{~Hz}$ in the simulations. Average speed of a carp ranges from 0 to $2.5 \mathrm{~m} / \mathrm{s}$ in the ichthyology. In the experimental studies, the speed range of forward swimming is recorded from $0.105 \mathrm{~m} / \mathrm{s}$ to $0.41 \mathrm{~m} / \mathrm{s}$ and turning speed is obtained as $0.24 \mathrm{~m} / \mathrm{s}$, approximately. It is also seen that the simulation speed values remained in these ranges. The pitch angle of the descending pattern of the carp is measured as $35^{\circ}$ during $1.05 \mathrm{~s}$. According to simulation results, the descending motion of the robotic fish is performed with $0.24 \mathrm{~s}$ settling time for $20^{\circ}$ pitch angle, which has very similar performance to carp.

Forward and turning speeds, descending-ascending motions and yaw, pitch, roll angles are also controlled to achieve real fish behaviors. Figures 11,17 and 18 show the maneuverability of the fish-like motions. Similarly, two different reference trajectories $\left(\mathrm{P}_{1}\right.$ and $\left.\mathrm{P}_{2}\right)$ are tracked to evaluate sufficient swimming performances in Figures 19 and 22. The proposed closed loop control system is also adapted to VRML in order to validate the $\mathrm{P}_{2}$ tracking in virtual marine environment. These simulation and 
experimental analyses show that the derived model achieves real carp motions to implement robotic fish prototypes for biomimetic design.

In future work, these experiences should be transferred to robotic fish prototypes in experimental platforms and path-tracking performance should be examined with various intelligent control algorithms.

Acknowledgments: This research was supported by the 114E652 TUBITAK 1001 project. We thank because of the financial support and guiding reports.

Author Contributions: Gonca Ozmen Koca, Cafer Bal, Deniz Korkmaz, Mustafa Can Bingol, Mustafa Ay, Zuhtu Hakan Akpolat and Seda Yetkin conceived all ideas and worked together to achieve this work.

Conflicts of Interest: The authors declare no conflict of interest.

\section{References}

1. Yu, J.; Wang, M.; Su, Z.; Tan, M.; Zhang, J. Dynamic modeling of a CPG-governed multijoint robotic fish. Adv. Robot. 2013, 27, 275-285. [CrossRef]

2. Ding, R.; Yu, J.; Yang, Q.; Tan, M.; Zhang, J. Dolphin-like swimming modeling for a biomimetic amphibious robot. IFAC Proc. Vol. 2011, 18, 9367-9372. [CrossRef]

3. Zhou, C.; Cao, Z.; Wang, S.; Tan, M. A marsupial robotic fish team: Design, motion and cooperation. Sci. China Technol. Sci. 2010, 53, 2896-2904. [CrossRef]

4. Lauder, G.V.; Madden, P.G.A. Learning from fish: Kinematics and experimental hydrodynamics for roboticists. Int. J. Autom. Comput. 2006, 3, 325-335. [CrossRef]

5. Zhang, F.; Tan, X. Three-Dimensional Spiral Tracking Control for Gliding Robotic Fish. In Proceedings of the 2014 IEEE 53rd Annual Conference on Decision and Control (CDC), Los Angeles, CA, USA, 15-17 December 2014; pp. 5340-5345.

6. Yang, G.H.; Choi, W.; Lee, S.H.; Kim, K.S.; Lee, H.J.; Choi, H.S.; Ryuh, Y.S. Control and Design of a 3 DOF Fish Robot 'ICHTUS'. In Proceedings of the 2011 IEEE International Conference on Robotics and Biomimetics (ROBIO), Karon Beach, Phuket, Thailand, 7-11 December 2011; pp. 2108-2113.

7. Zhou, C.; Tan, M.; Cao, Z.; Wang, S.; Creighton, D.; Gu, N.; Nahavandi, S. Kinematic Modeling of a Bio-Inspired Robotic Fish. In Proceedings of the IEEE International Conference on Robotics and Automation, Pasadena, CA, USA, 19-23 May 2008; pp. 695-699.

8. Suebsaiprom, P.; Lin, C.L. Fish-Tail Modeling for Fish Robot. In Proceedings of the 2012 International Symposium on Computer, Consumer and Control (IS3C), Taichung, Taiwan, 4-6 June 2012; pp. 548-551.

9. Ryuh, Y.S.; Yang, G.H.; Liu, J.; Hu, H. A school of robotic fish for mariculture monitoring in the sea coast. J. Bionic Eng. 2015, 12, 37-46. [CrossRef]

10. Masoomi, S.F.; Gutschmidt, S.; Chen, X.; Sellier, M. The Kinematics and Dynamics of Undulatory Motion of a Tuna-mimetic Robot. Int. J. Adv. Robot. Syst. 2015, 12, 83. [CrossRef]

11. Liu, J.; Hu, H. Biological Inspiration: From Carangiform Fish to Multi-Joint Robotic Fish. J. Bionic Eng. 2010, 7, 35-48. [CrossRef]

12. Niu, X.; Xu, J.; Ren, Q.; Wang, Q. Locomotion learning for an anguilliform robotic fish using central pattern generator approach. IEEE Trans. Ind. Electron. 2014, 61, 4780-4787. [CrossRef]

13. Li, G.; Deng, Y.; Osen, O.L.; Bi, S.; Zhang, H. A Bio-Inspired Swimming Robot for Marine Aquaculture Applications: From Concept-Design to Simulation. In Proceedings of the Oceans 2016-Shanghai, Shanghai, China, 10-13 April 2016; pp. 1-7.

14. Sfakiotakis, M. Review of Fish Swimming Modes for Aquatic Locomotion. IEEE J. Ocean. Eng. 1998, 24, 237-252. [CrossRef]

15. Suebsaiprom, P.; Lin, C.L. Maneuverability modeling and trajectory tracking for fish robot. Control Eng. Pract. 2015, 45, 22-36. [CrossRef]

16. Chowdhury, A.R.; Prasad, B.; Kumar, V.; Kumar, R.; Panda, S.K. Design, Modeling and Open-Loop Control of a BCF Mode Bio-Mimetic Robotic Fish. In Proceedings of the 2011 International Siberian Conference on Control and Communications (SIBCON), Krasnoyarsk, Russia, 15-16 September 2011; pp. 226-231.

17. Hu, Y.H.; Zhao, W.; Wang, L. Vision-Based Target Tracking and Collision Avoidance for Two Autonomous Robotic Fish. IEEE Trans. Ind. Electron. 2009, 56, 1401-1410. 
18. Yu, J.; Wang, S.; Tan, M. A simplified propulsive model of bio-mimetic robot fish and its realization. Robotica 2005, 23, 101-107. [CrossRef]

19. Yu, J.Z.; Liu, L.Z.; Tan, M. Three-dimensional dynamic modelling of robotic fish: simulations and experiments. Trans. Inst. Meas. Control 2008, 30, 239-258.

20. Wang, W.; Xie, G.; Shi, H. Dynamic Modeling of an Ostraciiform Robotic Fish Based on Angle of Attack Theory. In Proceedings of the 2014 International Joint Conference on Neural Networks (IJCNN), Beijing, China, 6-11 July 2014; pp. 3944-3949.

21. Li, G.; Zhang, H.; Zhang, J.; Bye, R.T. Development of adaptive locomotion of a caterpillar-like robot based on a sensory feedback CPG model. Adv. Robot. 2014, 28, 389-401. [CrossRef]

22. Li, G.; Zhang, H.; Li, W.; Hildre, H.P.; Zhang, J. Design of Neural Circuit for Sidewinding of Snake-Like Robots. In Proceedings of the 2014 IEEE International Conference on Information and Automation (ICIA), Hailar, China, 28-30 July 2014; pp. 333-338.

23. Ijspeert, A.J. Central pattern generators for locomotion control in animals and robots: A review. Neural Netw. 2008, 21, 642-653. [CrossRef] [PubMed]

24. Liu, J.; Hu, H. A 3D simulator for autonomous robotic fish. Int. J. Autom. Comput. 2004, 1, 42-50. [CrossRef]

25. Yu, J.Y.J.; Liu, L.L.L.; Wang, L.W.L. Dynamic Modeling of Robotic Fish Using Schiehien's Method. In Proceedings of the 2006 IEEE International Conference on Robotics and Biomimetics, Kunming, China, 17-20 December 2006; pp. 457-462.

26. Liu, L.; Yu, J.; Wang, L. Dynamic Modeling of Three-Dimensional Swimming for Biomimetic Robotic Fish. In Proceedings of the IEEE International Conference on Intelligent Robots and Systems, Beijing, China, 9-15 October 2006; pp. 3916-3921.

27. Zhou, C.; Cao, Z.; Wang, S.; Tan, M. The Dynamic Analysis of the Backward Swimming Mode for Biomimetic Carangiform Robotic Fish. In Proceedings of the IROS 2008 IEEE/RSJ International Conference on Intelligent Robots and Systems, Nice, France, 22-26 September 2008; pp. 3072-3076.

28. Nakashima, M.; Ohgishi, N.; Ono, K. A study on the propulsive mechanism of a double jointed fish robot utilizing self-excitation control. JSME Int. J. Ser. C Mech. Syst. Mach. Elem. Manuf. 2003, 46, 982-990. [CrossRef]

29. Kim, H.; Lee, B.; Kim, R. A Study on the motion mechanism of articulated fish robot. In Proceedings of the 2007 IEEE International Conference on Mechatronics and Automation (ICMA), Harbin, China, 5-8 August 2007; pp. 485-490.

30. Suebsaiprom, P.; Lin, C.L. 2-DOF Barycenter Mechanism for Stabilization of Fish-Robots. In Proceedings of the 2013 IEEE 8th Conference on Industrial Electronics and Applications (ICIEA), Melbourne, VIC, Australia, 19-21 June 2013; pp. 1119-1122.

31. Yu, J.; Wang, L.; Tan, M. Geometric optimization of relative link lengths for biomimetic robotic fish. IEEE Trans. Robot. 2007, 23, 382-386. [CrossRef]

32. Bal, C.; Korkmaz, D.; Koca, G.O.; Ay, M.; Akpolat, Z.H. Link Length Optimization of A Biomimetic Robotic Fish Based on Big Bang-Big Crunch Algorithm. In Proceedings of the 21st International Conference on Methods and Models in Automation and Robotics (MMAR), Miedzyzdroje, Poland, 29 August-1 September 2016; pp. 189-193.

33. Yu, J.; Tan, M.; Chen, J.; Zhang, J. A survey on CPG-inspired control models and system implementation. IEEE Trans. Neural Netw. Learn. Syst. 2014, 25, 441-456. [PubMed]

34. Ozmen Koca, G.; Korkmaz, D.; Bal, C.; Akpolat, Z.H.; Ay, M. Implementations of the route planning scenarios for the autonomous robotic fish with the optimized propulsion mechanism. Measurement 2016, 93, $232-242$. [CrossRef]

35. Vo, T.Q.; Kim, H.S.; Lee, B.R. Smooth gait optimization of a fish robot using the genetic-hill climbing algorithm. Robotica 2012, 30, 257-278. [CrossRef]

36. Gao, W.B.; Wang, Y.F.; Homaifa, A. Discrete-Time Variable-Structure Control-Systems. IEEE Trans. Ind. Electron. 1995, 42, 117-122.

37. Akpolat, Z.; Gokbulut, M. Discrete time adaptive reaching law speed control of electrical drives. Electr. Eng. 2003, 85, 53-58. [CrossRef]

38. Akpolat, Z.H.; Guldemir, H. Trajectory following sliding mode control of induction motors. Electr. Eng. 2003, 85, 205-209. [CrossRef] 
(C) 2018 by the authors. Licensee MDPI, Basel, Switzerland. This article is an open access article distributed under the terms and conditions of the Creative Commons Attribution (CC BY) license (http://creativecommons.org/licenses/by/4.0/). 